\title{
Influencia de la morfogénesis en la estabilidad de laderas
}

\author{
Influence of morphogenesis on the stability of slopes
}

Fecha de entrega: 14 de febrero 2019

Fecha de aceptación: 4 marzo 2020

\section{Juan Camilo Pineda y Carlos Eduardo Rodríguez}

Departamento de Ingeniería Civil, Universidad Nacional de Colombia, Carrera 30 No. 45-03, Bogotá D.C., Colombia, jucpinedasi@unal.edu.co, cerodriguezpi@unal.edu.co

La estabilidad de las laderas es definida por factores intrínsecos (geológicos, geomorfológicos, hidrogeológicos y geotécnicos) y exógenos (lluvias y sismos), que al analizarse $y$ relacionarse correctamente permiten comprender los procesos de inestabilidad y dar soluciones ingenieriles de intervención. Se evalúa la influencia de la morfogénesis, como componente de la geomorfología, en las condiciones de estabilidad mediante modelación numérica, analizando el estado de esfuerzos, el factor de seguridad y los mecanismos de falla para los ambientes estructural, denudaciones y fluvial. Al comparar los resultados con modelos numéricos de laderas en condición geostática se concluye que la magnitud y dirección de los esfuerzos en las laderas depende de los procesos de carga y descarga durante su origen.

Palabras clave: morfogénesis, procesos geomorfológicos, esfuerzos, factor de seguridad.
Slope stability is defined by intrinsic factors (geological, geomorphological, hydrogeological and geotechnical) and exogenous factors (rains and earthquakes), that when analyzed and correlated correctly allow to understand the instability processes of the slope to give engineering solutions of intervention. The influence of morphogenesis, as a component of geomorphology, is evaluated in the stability conditions with numerical models, analyzing the stress state, the safety factor and the failure mechanisms for the structural, denudation and fluvial environments. When comparing the results with numerical models of slopes in a geostatic condition, it is concluded that the magnitude and direction of the stress on the slopes depends on the loading and unloading processes during their origin.

Keywords: morphogenesis, geomorphological processes, stress, safety factor

\section{Introducción}

La geomorfología constituye una herramienta fundamental para poder evaluar y proyectar el comportamiento de los terrenos, y su interrelación con obras de infraestructura. A partir de la modelación numérica de los procesos de formación geomorfológicos, se puede evaluar su influencia en la estabilidad de las laderas representando el historial de esfuerzos generado durante la formación del relieve. Las diferentes geoformas son consecuencia de la interacción de la corteza con diferentes agentes geomorfológicos, que producen en el relieve procesos de carga y descarga que lo moldean. Los agentes y procesos geomorfológicos dependen de las condiciones geológicas y climáticas locales, características que son agrupadas en ambientes geomorfológicos. Se realiza una modelación numérica para los ambientes estructurales, denudacionales y fluviales en el programa de diferencias finitas FLAC 2D, aplicando modelos elastoplásticos, con una ley de plasticidad tipo Mohr-Coulomb, asociado con una ley de ablandamiento por deformación. A partir del análisis y validación de los resultados de los modelos de procesos geomorfológicos, se estudia una ladera en particular, comparando los resultados con un modelo de una ladera de igual geoforma pero en condición geostática. Los parámetros de análisis y comparación son: el factor de seguridad FS y la magnitud y dirección de los esfuerzos.

\section{Modelaciones previas}

Los investigadores han utilizado varios modelos numéricos para analizar la evolución tectónica de las zonas de subducción oceánica que buscan representar el empuje de la litosfera, de la corteza inferior, de la corteza superior 
y en una segunda fase el cabalgamiento entre placas de la corteza superior. La reología comúnmente utilizada es un modelo viscoso dependiente de la temperatura, y, en menor manera, modelos elástico-plásticos (e.g. Sobolev y Babeyko, 2005). Las modelaciones realizadas por Platt (1986) y por Willet et al. (1993) utilizaban modelos de cinturones de empuje y plegado y cuñas de acreción, asumiendo una deformación friccional-frágil modelados con reologías perfectamente plásticas o con un criterio de falla tipo Mohr-Coulomb. Posteriormente los modelos fueron desarrollados para explicar la evolución completa del proceso orogénico y las capas que lo componen (litosfera, corteza superior e inferior), en los cuales fue necesario incluir reologías que representaran las deformaciones dúctiles que controlan el comportamiento de las capas viscosas.

Los procesos superficiales de erosión y sedimentación no son considerados en la mayoría de las investigaciones, debido a que estos procesos no impactan significativamente en la formación y propagación de las zonas de corte, pero pueden influir significativamente en su orientación, espaciamiento y evolución (Jaquet et al., 2018). Jaquet et al. (2018) elaboraron un modelo de elementos finitos con comportamiento viscoplástico dependiente de la temperatura con una función de re-enmallado cada vez que un ángulo de cualquier elemento triangular fuera menor de $30.5^{\circ}$. El modelo consta de un ancho de 1600 $\mathrm{km}$ y un espesor de $125 \mathrm{~km}$ incluyendo $20 \mathrm{~km}$ de corteza superior, $15 \mathrm{~km}$ de corteza inferior y $90 \mathrm{~km}$ de manto. Se realiza el acortamiento lateral, por medio de la aplicación de un desplazamiento en ambos costados, y se permite el deslizamiento en la frontera inferior. Ellos concluyen que los esfuerzos generados durante el equilibrio isostático no son relevantes al compararlos con los esfuerzos compresionales generados por el acortamiento de la litosfera.

Para este ambiente de formación, se han realizado modelaciones a menores escalas espaciales, localizadas en zonas de estudio específicas y de espesores del orden de 3 a $5 \mathrm{~km}$. Estos son conocidos como modelos de empuje tectónico o modelos de plegamiento por empuje, los cuales representan la compresión de las placas tectónicas fuera de la zona de subducción u obducción, donde se presenta la aplicación de esfuerzos de compresión a una escala menor, en las zonas llamadas cinturones de plegado y empuje (fold-and-thrustbelts). Buiter et al. (2006) realizaron una modelación de un empuje lateral en un material frágil y la compararon con varias modelaciones análogas tipo caja de arena (sandbox). En las modelaciones numéricas y en los modelos análogos de comparación, no se evalúa la influencia de la variación de la rigidez de los estratos. La estratificación inicial corresponde a un mismo material granular, con mismas propiedades de elasticidad y de resistencia diferenciado en capas por cambio de color únicamente. Por otro lado, Ellis et al. (2004) propone un modelo de acortamiento considerando una variación de resistencia, tomando ángulos de fricción interna con diferencias del orden de $1^{\circ}$ a $2^{\circ}$. Este modelo de empuje tectónico se compara con un modelo análogo, donde se hace un análisis detallado de la escala y correspondencia entre los parámetros de ambos modelos para finalmente comparar las secuencias de deformación. Para el modelo análogo $1 \mathrm{~km}$ corresponde a $1 \mathrm{~cm}$; y la velocidad de 1 $\mathrm{cm} /$ año corresponde a una velocidad de $4.8 \mathrm{~cm} /$ hora. Se considera un ángulo de fricción entre la base y las paredes con el material de $28^{\circ}$.

Un modelo de empuje tectónico muy similar fue propuesto por Barnichon (1998), utilizando un material granular, investigó la influencia de la variación de fricción en la base, simulando un modelo análogo desarrollado por Colletta et al. (1991). La longitud del modelo es de $20 \mathrm{~cm}$ y un espesor de $1.4 \mathrm{~cm}$. Se realizó el empuje en el costado izquierdo del modelo en 5 fases de desplazamiento: $0.3 \mathrm{~cm}, 1.5 \mathrm{~cm}$, $2.45 \mathrm{~cm} 3.36 \mathrm{~cm}$ y $4.5 \mathrm{~cm}$; y se varío la fricción en la base del modelo de $15^{\circ}$ a $25^{\circ}$. Ruh et al. (2012) concluyeron que el espesor de la corteza (del modelo) influye también en la generación y separación de las cuñas y zonas de falla. También estudiaron la influencia de la presencia de estratos de menor resistencia durante los procesos de carga tectónica, especialmente en la base del modelo, simulando el deslizamiento de estratos rocosos sobre otros en una zona de falla llamada décollement o falla de desprendimiento basal, para este caso de estudio, formada sobre lutitas saturadas o estratos de sal viscosa. La distribución de los estratos de menor resistencia condicionan de manera significativa la evolución estructural de la corteza y la deformación topográfica del relieve; ya que los estratos de menor resistencia favorecen el plegado por encima del fallamiento porque favorecen al deslizamiento flexural (deslizamiento entre los estratos al plegarse). El modelo de Zhang (2014) permite el desplazamiento de la base 
del modelo en dirección vertical, dando como resultado deformaciones de levantamiento. Otra forma de modelar los procesos de compresión tectónica, es restringir el desplazamiento horizontal en la base, generalmente hasta la mitad del modelo, o incorporando al modelo una superficie de falla para simular el levantamiento topográfico en zonas de falla inversa o de cabalgamiento (Ford, 2015).

La mayoría de las investigaciones han usado modelos elásto-visco-plásticos con viscosidad dependiente de la temperatura y parámetros de resistencia dependientes de la condición de esfuerzos. Para esta investigación, se proponen modelos elasto-visco-plásticos con parámetros reológicos que no dependen de la temperatura porque la zona de análisis es en la superficie de la corteza. Aunque se realizan modelaciones de escala mayor (modelos de hasta $100 \mathrm{~km}$ de profundidad), la condición de esfuerzos que se evalúa es en la parte superficial, donde la temperatura es tan baja que la viscosidad es muy alta y se comporta como un material con reología elásto-plástica tipo Coulomb.

En este trabajo se presenta una modelación numérica para los ambientes estructurales, denudacionales y fluviales en el programa de diferencias finitas FLAC 2D (2011), aplicando modelos elasto-plásticos, con una ley de plasticidad tipo Mohr-Coulomb, asociado con una ley de ablandamiento por deformación. A partir del análisis y validación de los resultados de los modelos de procesos geomorfológicos, se estudia una ladera en particular, comparando los resultados con un modelo de una ladera de igual geoforma pero en condición geostática. Los parámetros de análisis y comparación son: el factor de seguridad FS y la magnitud y dirección de los esfuerzos.

\section{Modelo numérico}

Las modelaciones se realizaron en dos dimensiones, utilizando el método por diferencias finitas para simular el comportamiento de los suelos y rocas. El método consiste en el remplazo de las derivadas de las ecuaciones que gobiernan el comportamiento del suelo por expresiones algebraicas escritas en términos de las variables de campo (esfuerzo versus deformación) para puntos discretos en el espacio.

\section{Modelo constitutivo}

Se considera que un material presenta un comportamiento plástico cuando está sometido a una carga que produce

deformaciones irreversibles y permanentes. Para estimar

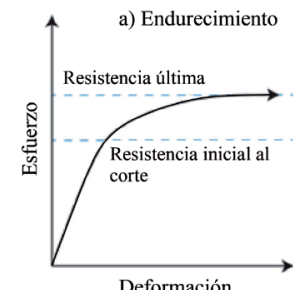

Deformación

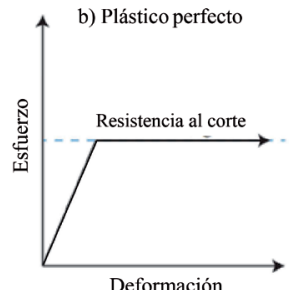

Deformación

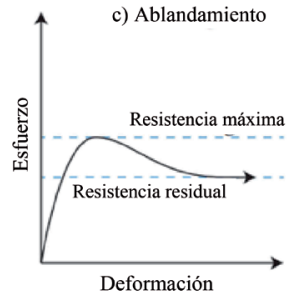

Deformación

Figura 1: Relación esfuerzo-deformación para modelos elastoplásticos (Sørensen, 2012)

\section{Re-enmallado}

Las modelaciones de procesos geomorfológicos son de altas deformaciones y en la mayoría los elementos de la malla presentan altas distorsiones, adicionalmente, estas requieren continuidad de desplazamientos aun después de alcanzar el estado plástico en algunas zonas. Para modelar estas condiciones, es necesario generar una función que, al momento de alcanzar la distorsión de algún elemento, automáticamente genere una nueva malla, zonificando los esfuerzos, velocidades y desplazamientos y de continuidad al análisis. Este tipo de códigos son implementados en los modelos de Ellis (2004), Ruh et al. (2012) y Jaquet et al. (2018).

\section{las deformaciones plásticas es necesario utilizar la teoría de la plasticidad y modelos elastoplásticos formulados en la mecánica del medio continuo. Los diferentes modelos constitutivos se caracterizan por su función de fluencia, función de endurecimiento/ablandamiento por deformación y su ley de flujo. El modelo constitutivo de fluencia empleado para las modelaciones es el de Mohr-Coulomb. Para un modelo elastoplástico perfecto, la superficie de fluencia no varía cuando es alcanzada por los esfuerzos del material, en cambio para los modelos elastoplásticos de ablandamiento/endurecimiento por deformación, esta no es constante, sino crece o decrece según el modelo como se presenta en la Figura 1. Al modelo constitutivo utilizado se le asocia una ley de ablandamiento por deformación, en la cual la resistencia del material disminuye a medida que se presentan deformaciones plásticas hasta llegar a la resistencia residual.

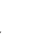

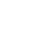


las recomendaciones dadas por Han (2008) para procesos tectónicos. En la Figura 2 se ejemplifica como para un escenario de empuje tectónico, una parte de la malla alcanza una alta distorsión, momento en el cual se genera la rezonificación del modelo teniendo una malla mejor distribuida y conservando la misma condición de esfuerzos.

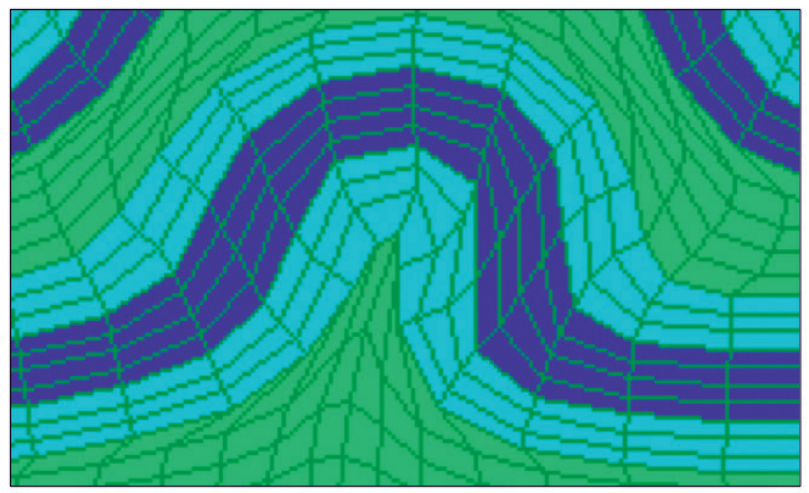

Figura 2: Distorsión de una parte de la malla antes de re-enmallar

\section{Modelación numérica}

La Figura 3 presenta el proceso de análisis seguido para el desarrollo de las modelaciones. El paso 1 hace referencia a la revisión y análisis de la bibliografía necesaria para la construcción de los modelos, se define su geometría, estratigrafía y propiedades geomecánicas. En esta etapa del proceso hay mayores incertidumbres relacionadas a la recolección de la información de entrada y en la certeza de las hipótesis asumidas.

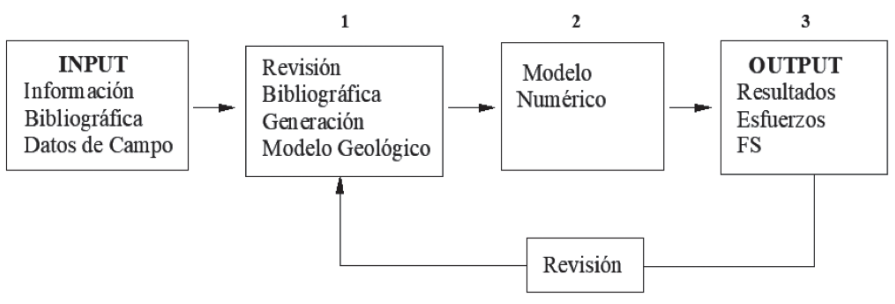

Figura 3: Esquema del proceso de modelación

El paso 2 es la generación del modelo numérico en el software FLAC 2D (2011), ingresando los parámetros determinados anteriormente y estructurando las fases de análisis. En el paso 3 se analizan los resultados de la modelación, donde su validez depende de la efectividad de los pasos anteriores. En caso de que los resultados no presenten congruencia con los conceptos teóricos de la bibliografía o se presenten errores de convergencia, se deberá volver al paso 1 y verificar los datos ingresados.

Se realiza la modelación de los siguientes ambientes geomorfológicos: estructural (compresión y extensión), denudacional (erosión y fluvial) y un modelo de fases progresivas.

Las escalas temporales se analizan en tasas de desplazamiento y en tasas de erosión. No se considera la estratigrafía detallada ni las fallas geológicas menores. Siguiendo estos criterios se estima que los modelos representarán a gran escala los procesos de carga que se desarrollan durante la morfogénesis, permitiendo observar los cambios en la distribución de esfuerzos en las laderas.

Los resultados son analizados en tres fases. Primero se analiza para la placa tectónica en general la magnitud y la dirección de los esfuerzos principales, el máximo incremento de la deformación cortante y las zonas de plasticidad. En la segunda fase se analiza, para la zona específica de la ladera, la condición de esfuerzos principales y el FS utilizando la metodología de reducción de parámetros de resistencia. Finalmente, se comparan los resultados obtenidos con un modelo de condición de esfuerzos geostática (convencional).

\section{Ambiente estructural Procesos de compresión}

Los procesos de carga a compresión generados en un ambiente estructural se analizaron en tres escenarios para diferentes escalas y condiciones de frontera, con el objetivo de simular lo más aproximado las condiciones de tectonismo reales.

- Proceso de subducción

Se modela el proceso de subducción entre la corteza oceánica y la corteza continental responsable de levantamientos topográficos de mayor magnitud. Este consiste en el hundimiento de la corteza oceánica por debajo de la corteza continental, generando una alta presión de contacto entre ambas placas produciendo grandes esfuerzos, fallas y deformaciones. En la Figura 4 se presentan las dimensiones del modelo y los parámetros de los materiales adoptados para el modelo según los criterios de modelación propuestos por Pasupuleti (2016), Jaquet et al. (2018), Platt (1986), Sobolev y Babeyko (2005), Willet et al. (1993) y Yamato et al. (2009). 


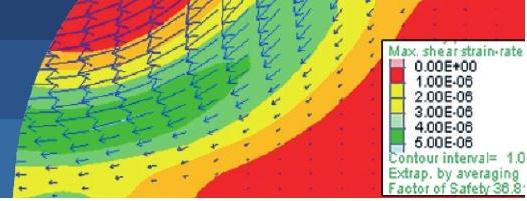

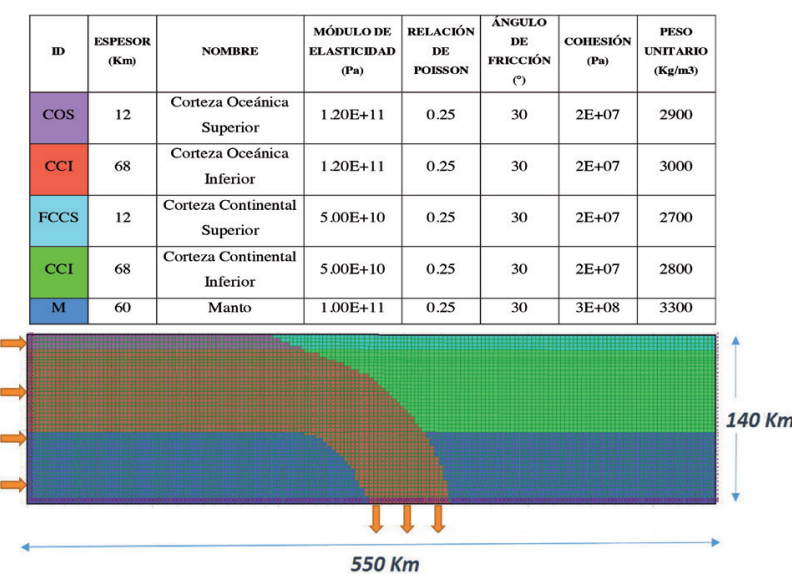

Figura 4: Modelo escenario de subducción

Para validar el modelo se revisaron los resultados para un desplazamiento horizontal de 3, 18 y $34.7 \mathrm{~km}$. En la Figura 5 se presentan las máximas tasas de deformación cortante en la fase final de $34.7 \mathrm{~km}$, indicando las zonas de falla por cortante y de desplazamiento plástico. La orientación del esfuerzo principal es horizontal en la corteza que no presenta falla y vertical en las cuñas de falla.

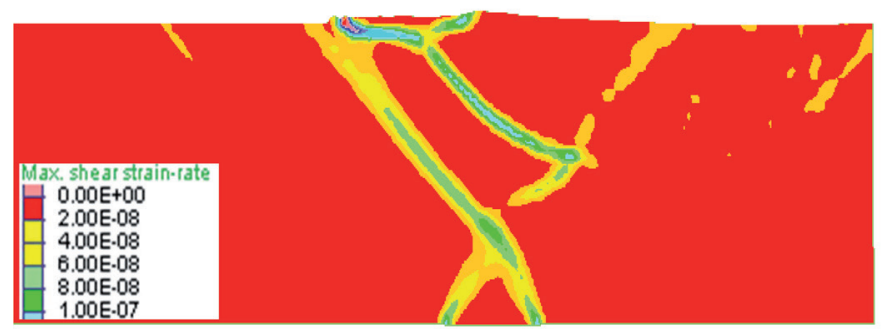

Figura 5: Zonas de máxima tasa de deformación cortante. Desplazamiento lateral de $34694 \mathrm{~m}$

En la Figura 6 se comparan los resultados numéricos obtenidos de las zonas de falla (color rosa) con las propuestas por Nemcoc et al. (2013) teniendo resultados consistentes.

a)

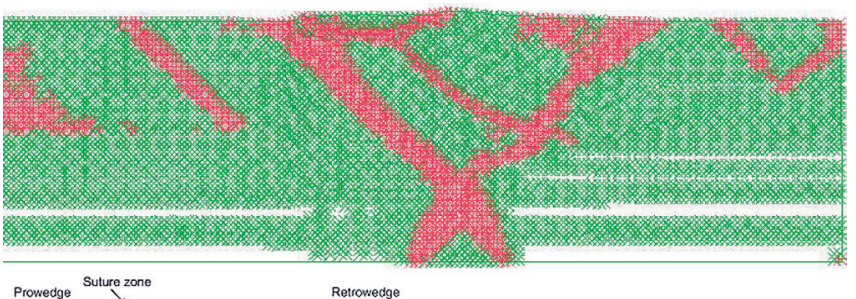

b)
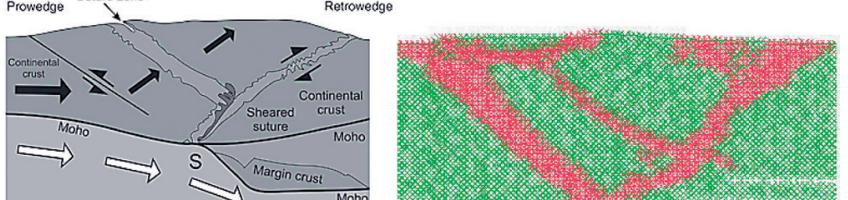

Figura 6: a) Zonas de plastificación: resultado FLAC 2D y b) comparación con zonas propuestas por Nemcoc et al. (2013)
En la zona donde subyace la corteza oceánica, el modelo representó el levantamiento topográfico de una cadena montañosa, comparable con la cordillera de los Andes, como se observa en la Figura 7. Se presenta la distribución y magnitud del esfuerzo principal mayor, el cual tiene una orientación horizontal en las placas que colisionan y vertical en la superficie de la corteza continental, en la zona posterior a la subducción.

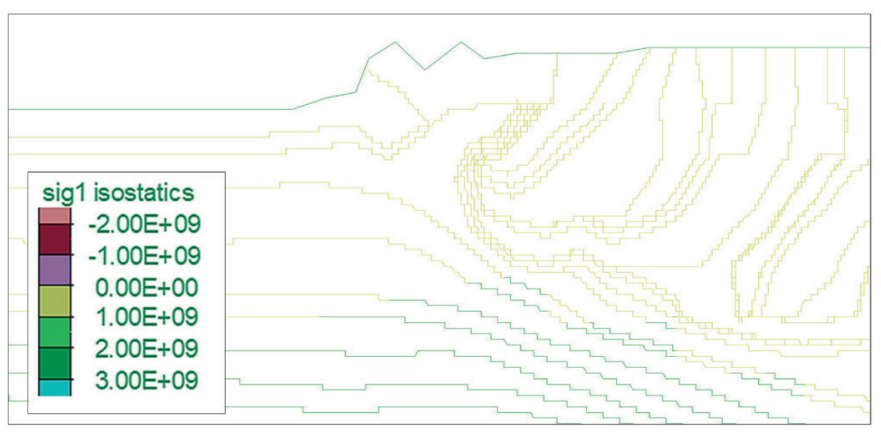

Figura 7: Levantamiento del relieve en zona de subducción

Debido a la escala de magnitud del modelo, los elementos de la malla presentan grandes dimensiones en relación a los demás modelos siendo una limitación para el análisis detallado de la ladera.

- Empuje tectónico-plegamiento

Se modela un empuje tectónico donde los esfuerzos globales son compresivos y el esfuerzo principal mayor está orientado horizontalmente, ambiente en el cual se forman las cadenas montañosas. El perfil estratigráfico utilizado se presenta en la Figura 8. Las dimensiones, estratificación y parámetros de este modelo se trabajaron a partir de las recomendaciones y modelaciones realizadas por Zhang (2014). Barnichon (1998) concluyó que el cambio de rigideces y resistencias en el perfil estratigráfico influye significativamente en la magnitud de los esfuerzos

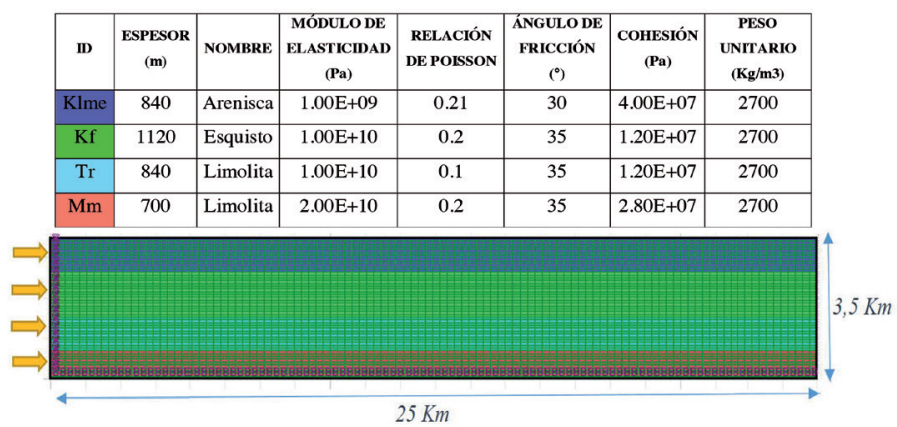

Figura 8: Modelo de empuje tectónico-plegamiento 
durante este proceso, por lo cual, se consideraron estratos con parámetros de resistencia y densidades diferentes. En el estrato basal del modelo se considera una resistencia mucho menor a los demás, siendo netamente friccionante, siguiendo el criterio de que el deslizamiento se genera en un estrato débil según Ruh et al. (2012).

En la Figura 9 se presenta la tasa máxima del incremento de deformación cortante para un desplazamiento de 2, 2.5 y $3 \mathrm{~km}$. Se observa como se genera la propagación de la superficie de falla y el desplazamiento de la cuña dando forma al relieve. Esta deformación es consistente con la deformación progresiva del relieve que ilustra Barnichon (1998) y los ángulos de falla y deformaciones topográficas son similares a los modelos de Ellis et al. (2004).
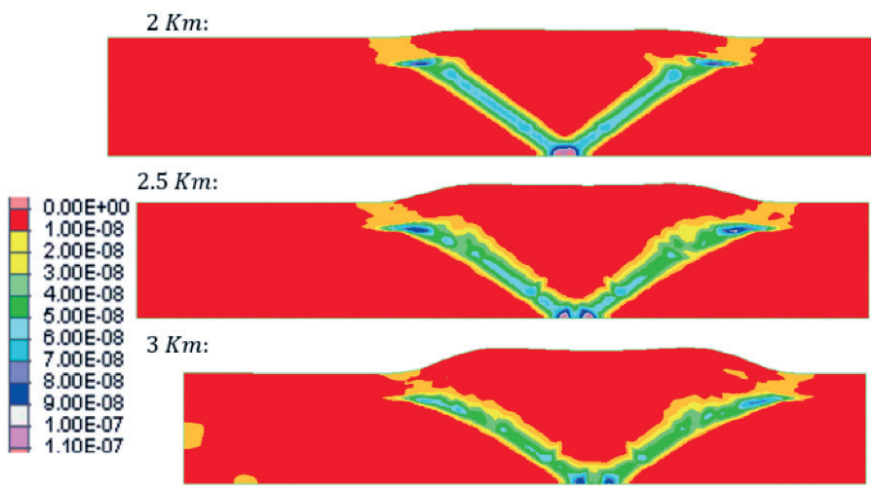

Figura 9: Máxima tasa de deformación cortante para 2, 2.5 y 3 $\mathrm{km}$ de desplazamiento

Se analiza en detalle la ladera generada por el levantamiento topográfico, de $840 \mathrm{~m}$ de altura y una longitud de 3000 $\mathrm{m}$ y se compara con un modelo de mismas condiciones litológicas y topográficas, pero sin considerar la trayectoria de los esfuerzos durante la morfogénesis. Se calculó FS para ambos modelos obteniendo 18.78 para el escenario morfogenético y de 60 para el escenario geoestático, indicando que el escenario que considera la morfogénesis está más cercano a fallar. Sin embargo, los altos factores de seguridad y las tasas de deformación cortante presentadas en la Figura 10, muestran que éste no es un parámetro representativo de comparación ya que se están obteniendo superficies de falla de gran profundidad y por las condiciones de modelación no se representan las fallas a menor profundidad, que son las que se evidencian en campo.

En la Figura 11 se presentan la distribución y magnitud a)

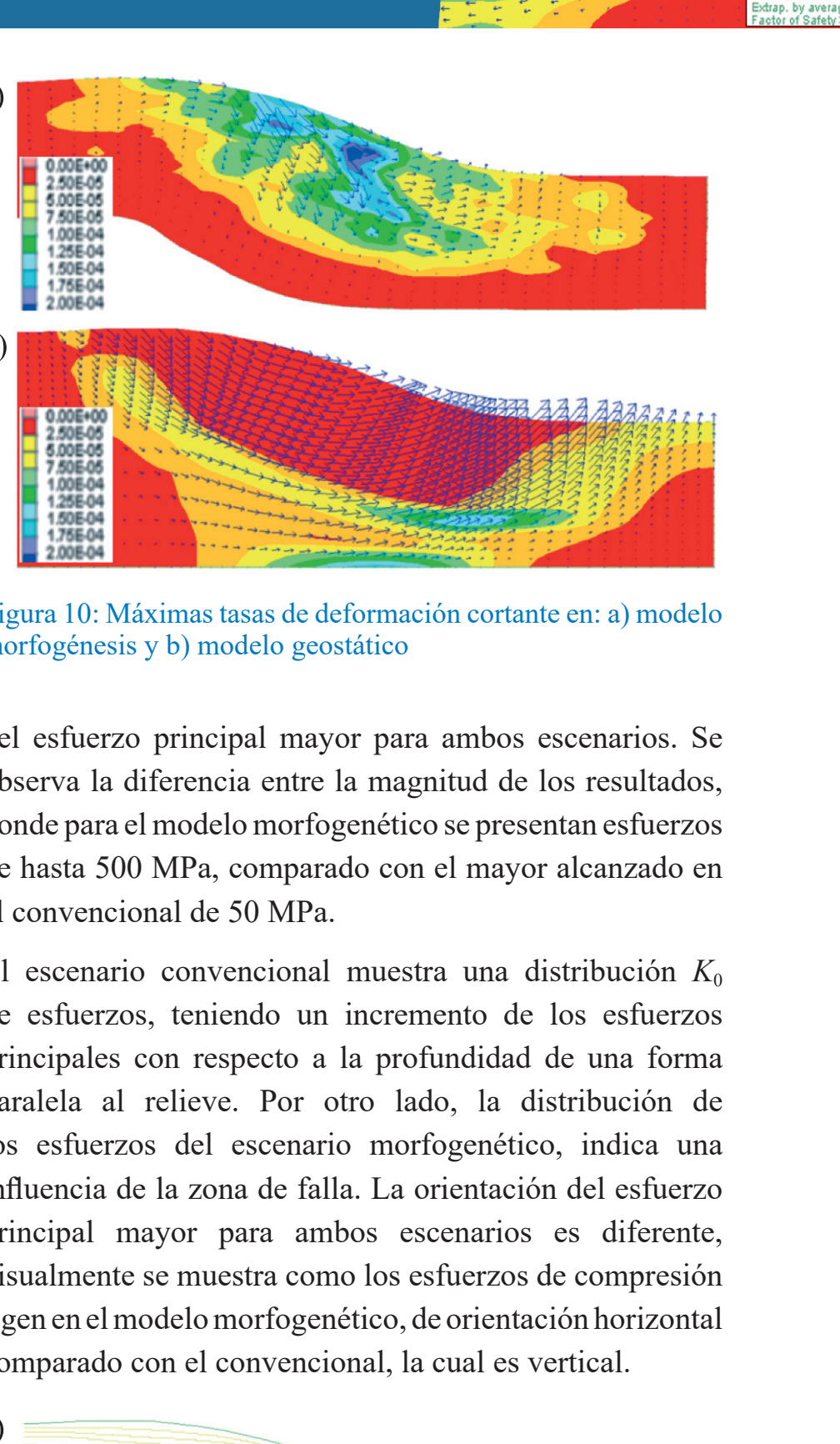

b)

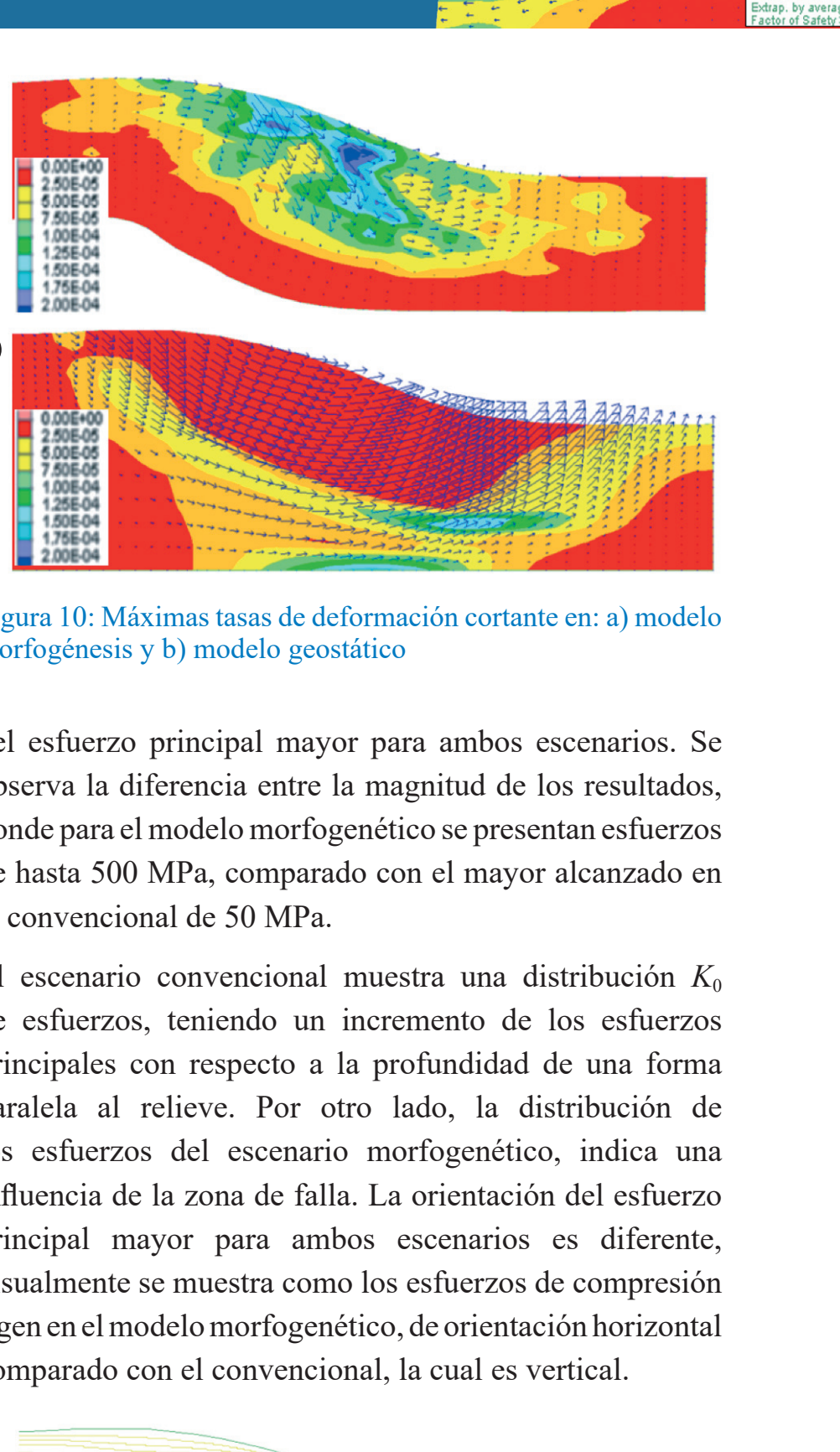

Figura 10: Máximas tasas de deformación cortante en: a) modelo morfogénesis y b) modelo geostático

del esfuerzo principal mayor para ambos escenarios. Se observa la diferencia entre la magnitud de los resultados, donde para el modelo morfogenético se presentan esfuerzos de hasta $500 \mathrm{MPa}$, comparado con el mayor alcanzado en el convencional de $50 \mathrm{MPa}$.

El escenario convencional muestra una distribución $K_{0}$ de esfuerzos, teniendo un incremento de los esfuerzos principales con respecto a la profundidad de una forma paralela al relieve. Por otro lado, la distribución de los esfuerzos del escenario morfogenético, indica una influencia de la zona de falla. La orientación del esfuerzo principal mayor para ambos escenarios es diferente, visualmente se muestra como los esfuerzos de compresión rigen en el modelo morfogenético, de orientación horizontal comparado con el convencional, la cual es vertical.

a)

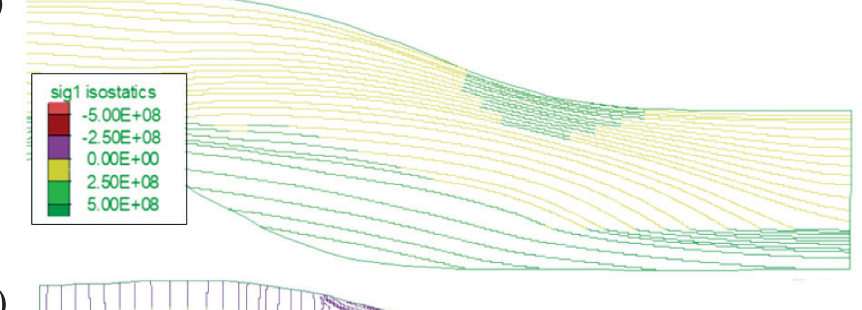

b)

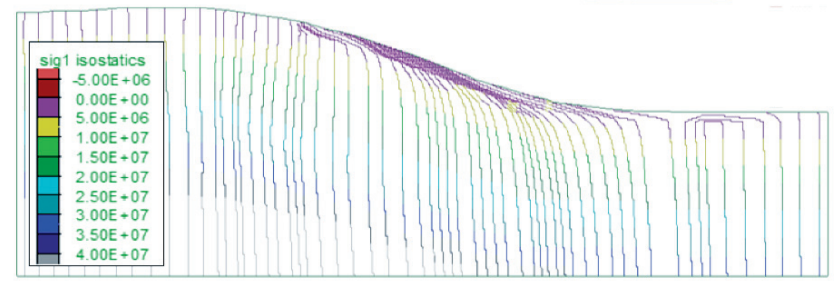

Figura 11: Esfuerzo principal mayor: a) modelo morfogénesis y b) modelo geostático 
- Empuje tectónico-cabalgamiento

Se realiza un modelo que represente las zonas de falla inversas donde se producen cabalgamientos entre placas. En este ambiente de formación se producen esfuerzos compresivos que se concentran principalmente en la placa cabalgante y en la zona de contacto. En la zona de flexión de la corteza se asocian esfuerzos de extensión. Se elaboró el modelo a partir del estudio realizado por Ford (2015), quien también implementó el programa Flac 2D, simulando la deformación del relieve en una zona de falla inversa. Se revisaron los criterios de modelación, la estratificación y los parámetros geomecánicos implementados en su investigación y se planteó el perfil estratigráfico de la Figura 12.

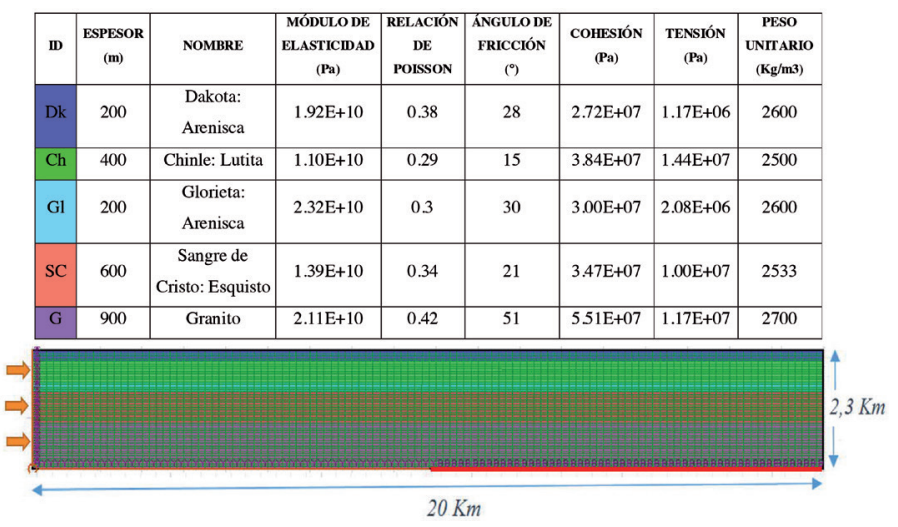

Figura 12: Modelo empuje tectónico-cabalgamiento

A diferencia del modelo de empuje tectónico anterior, se restringe el desplazamiento lateral en la base en la mitad del modelo para generar el cabalgamiento. En la Figura 13 se muestra en color rosa las zonas de plastificación donde se detallan las superficies de falla. La dirección del esfuerzo principal mayor es horizontal presentado una condición de compresión en el material.

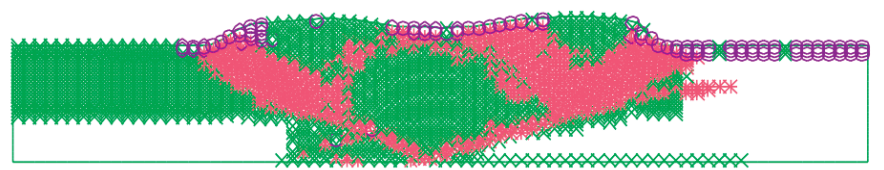

Figura 13: Zonas de plastificación para un acortamiento de $3 \mathrm{~km}$

Se analiza en detalle la ladera de $400 \mathrm{~m}$ de altura que está en el frente de avance de la falla. La geoforma resultante es de alta pendiente, susceptible a presentar mecanismos de falla de menor profundidad tipo caída de rocas y volcamientos. El FS obtenido para el escenario morfogenético fue de 1.54 y para el convencional de 18.65. Nuevamente se observa una gran diferencia entre los resultados, particularmente el escenario convencional presenta un valor relativamente alto.

En la Figura 14 se presenta la tasa de deformación cortante y los vectores de velocidad, se interpreta el posible mecanismo de falla a partir de estos resultados. Para el escenario convencional, el mecanismo resultante es de tipo rotacional mientras que para el morfogenético se presenta un levantamiento en la pata de la ladera, asociado al cabalgamiento de la cuña de falla.

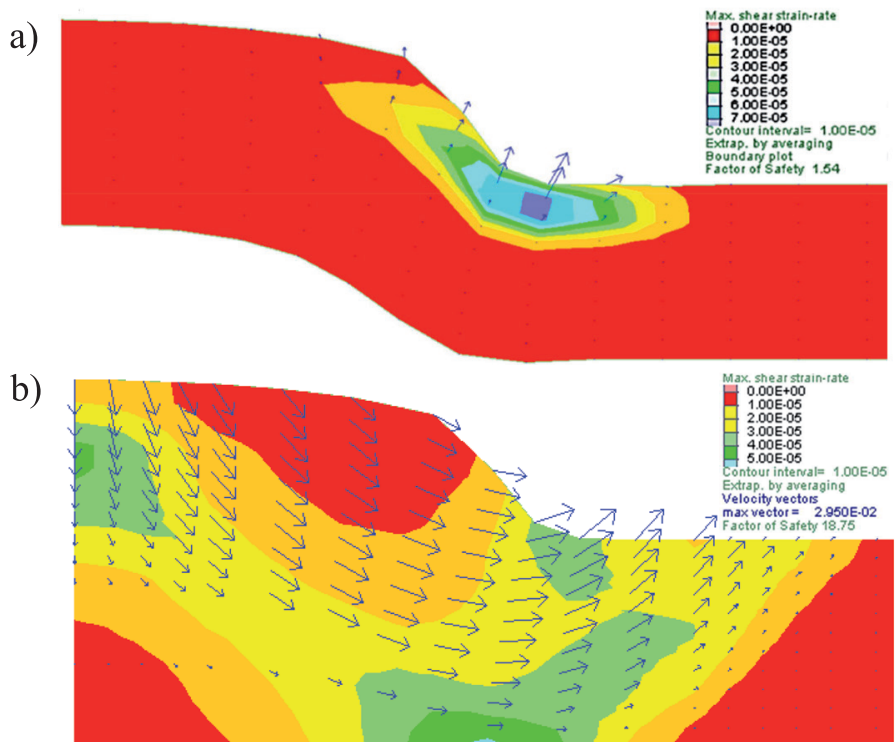

Figura 14: Máxima tasa de deformación cortante en: a) modelo morfogénesis y b) modelo geostático

En la Figura 15 se presenta el esfuerzo principal mayor resultante en ambas modelaciones, donde se observan diferencias significativas en magnitud y dirección especialmente en las zonas de falla. Para el escenario convencional, el esfuerzo incrementa con la profundidad paralelamente a la superficie del relieve; en cambio, para el escenario morfogenético, el incremento varía con respecto a la cercanía de la zona de falla. La orientación del esfuerzo principal mayor es horizontal para el escenario morfogenético mostrando un ambiente compresional, a diferencia del convencional donde es vertical. En el escenario morfogenético, la dirección de los esfuerzos principales en la corona y en la pata de la ladera presentan un comportamiento diferente al que se presenta en el resto del modelo (dirección paralela a la superficie topográfica). Esto se asocia a que el plegamiento y la fuerte pendiente de la ladera genera, en su corona, la tendencia a esfuerzos de tensión. 
a)

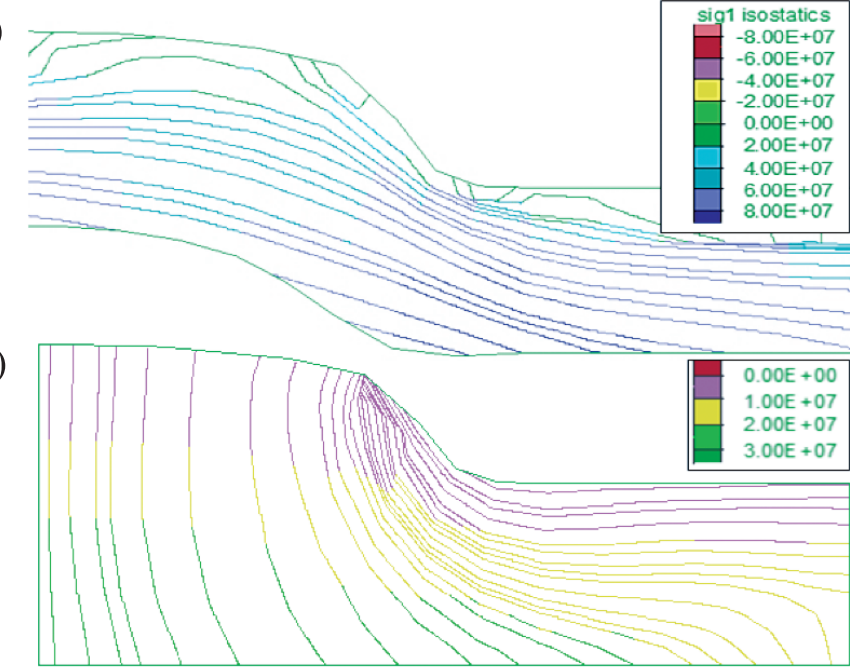

Figura 15: Esfuerzo principal mayor: a) modelo morfogénesis y b) modelo geostático

\section{Procesos de extensión}

Los escenarios de extensión tectónica son zonas de divergencia de la corteza terrestre producto de la separación de las placas tectónicas, como los rift continentales, valles de extensión, entre otros.

- Valle de extensión-graben

Se realiza la modelación de un valle de extensión, en el cual los esfuerzos debido al tectonismo son principalmente de tensión. Se tomaron como base las investigaciones realizadas por Buitter (2006) y Nagel y Buck (2004). Las dimensiones y parámetros del modelo se presentan en la Figura 16 donde se consideró un espesor de corteza de 35 $\mathrm{km}$ y un largo de $25 \mathrm{~km}$. El perfil estratigráfico se planteó considerando los modelos propuestos por Sclater y Célérier (1987) y Henk y Nemcok (2008), quienes modelan un escenario de extensión tipo graben.

\begin{tabular}{|c|c|c|c|c|c|c|c|c|}
\hline ID & $\begin{array}{c}\text { ESPESOR } \\
(\mathbf{m})\end{array}$ & NOMBRE & \begin{tabular}{|c|} 
MODULO DE \\
ELASTICIDAD \\
(Pa)
\end{tabular} & $\begin{array}{c}\text { RELACIÓN } \\
\text { DE } \\
\text { POISSON }\end{array}$ & $\begin{array}{l}\text { ÁNGULO DE } \\
\text { FRICCIÓN } \\
\left(^{\circ}\right)\end{array}$ & $\underset{(\mathbf{P a})}{\text { COHESIÓN }}$ & $\begin{array}{c}\text { TENSIÓN } \\
(\mathbf{P a})\end{array}$ & \begin{tabular}{|c} 
PESO \\
UNITARIO \\
(Kg/m3)
\end{tabular} \\
\hline $\mathrm{Lm}$ & 500 & Limolita & $5.37 \mathrm{E} 10$ & 0.26 & 27 & $2.68 \mathrm{E} 7$ & $1.58 \mathrm{E}+06$ & 2720 \\
\hline Ar & 200 & Arenisca & $2.55 \mathrm{E} 10$ & 0.38 & 28 & $2.7 \mathrm{E} 7$ & $1.17 \mathrm{E}+07$ & 2180 \\
\hline Gr & 1800 & Granito & 3.67E10 & 0.18 & 50 & $2.7 \mathrm{E} 7$ & $1.17 \mathrm{E}+07$ & 2640 \\
\hline sh & 350 & Shale & 3.67Eg & 0.25 & 10 & $7 \mathrm{E} 5$ & $1.17 \mathrm{E}+07$ & 2470 \\
\hline
\end{tabular}

Figura 16: Modelo valle de extensión-graben

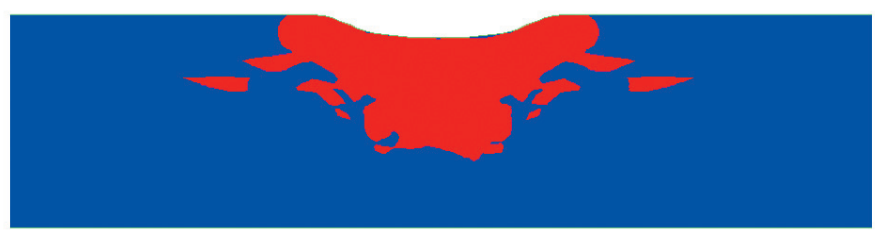

Figura 17: Zonas de compresión (azul) y tensión (rojo)

La condición de esfuerzos resultantes se presenta en la Figura 17 en la cual se observa las zonas donde los esfuerzos de tensión son predominantes (color rojo), principalmente en las laderas. La dirección del esfuerzo principal mayor presenta una orientación vertical y el esfuerzo principal menor es negativo en las zonas de tensión.

Este modelo es controlado por esfuerzos de tensión que generan fallas normales e influencian la dirección y magnitud de los esfuerzos principales. Los resultados se diferencian con los modelos tectónicos de compresión (que hacen parte del mismo ambiente geomorfológico) en que los esfuerzos de extensión rigen para el desarrollo de la geoforma.

Se realiza la modelación del escenario convencional y se comparan los factores de seguridad presentados en la Figura 18, donde se obtuvo 36.8 para el escenario morfogenético y 37.3 para el escenario geostático. Estos resultados son bastante similares, a diferencia de los resultados de los escenarios de empuje tectónico analizados previamente. Se concluye que, para el mecanismo de falla resultante la estabilidad global es similar. Sin embargo, al tener valores tan altos se estima de que otros tipos de falla puedan ocurrir y no se estén representando en el modelo por diversos factores tales como la densidad de la malla, el modelo constitutivo utilizado, la variabilidad de parámetros de resistencia, entre otros.

En la Figura 19 se presenta la condición del esfuerzo principal menor para ambos escenarios que tiene una orientación horizontal. La diferencia principal son las magnitudes obtenidas, donde para varias zonas del modelo morfogenético, el proceso de extensión reduce su magnitud, inclusive alcanzando valores negativos (esfuerzos de tensión). Adicionalmente, se observan diferencias en la dirección de los esfuerzos, especialmente en las zonas de falla, en la cual hay una rotación de los esfuerzos para el escenario morfogenético. 
a)

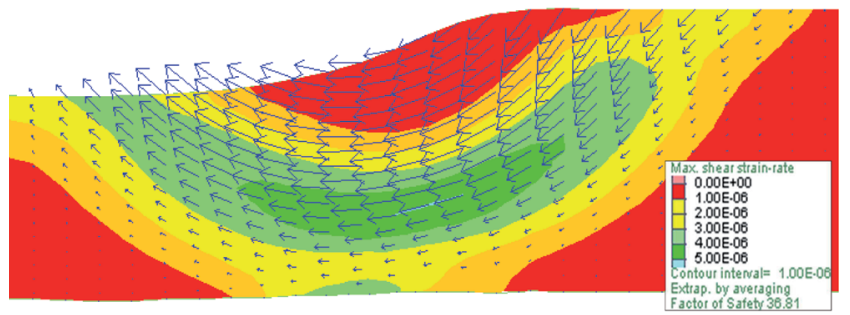

b)

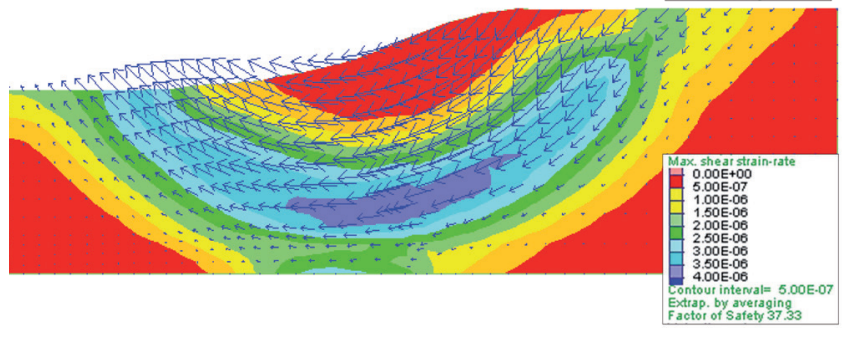

Figura 18: Máxima tasa de deformación cortante en: a) modelo morfogénesis y b) modelo geoestático

a)

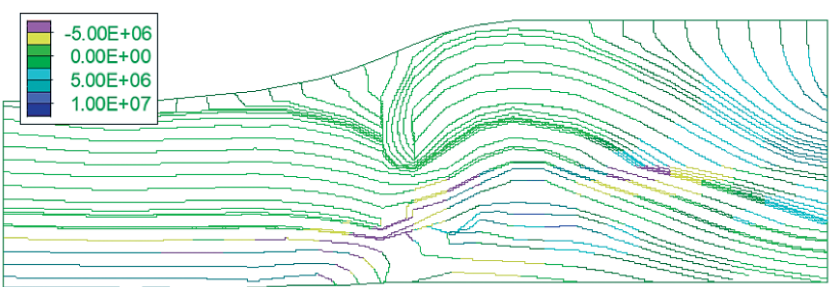

b)

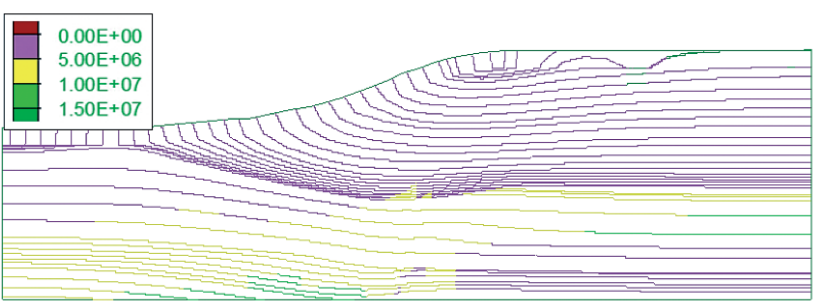

Figura 19: Esfuerzo principal menor: a) modelo morfogénesis y b) modelo geostático

\section{Ambiente denudacional}

\section{Procesos de erosión}

Debido a eventos climáticos, la superficie de la tierra es sometida a diferentes tipos de alteración, los cuales, sumados a la acción de la gravedad, generan la erosión del material. La morfometría del relieve es altamente influenciada por estos procesos debido a la intensidad y continuidad con que actúan, los cuales se acumulan y actúan simultáneamente junto con otros procesos geomorfológicos (tectónicos y fluviales). Los ambientes donde las geoformas resultantes son el resultado de la acción intensa de estos procesos de erosión se denominan denudacionales. Se realiza la modelación con base a la propuesta esquemática de Abbasi (2014), quien simula los procesos de compresión tectónica y de erosión de manera simultánea. En la Figura 20 se presentan los parámetros y dimensiones del modelo.

\begin{tabular}{|c|c|c|c|c|c|c|c|c|}
\hline ID & $\begin{array}{c}\text { ESPESOR } \\
(\mathrm{m})\end{array}$ & NOMBRE & $\begin{array}{l}\text { MODULO DE } \\
\text { ELASTICIDAD } \\
\text { (Pa) }\end{array}$ & $\begin{array}{c}\text { RELACIÓN } \\
\text { DE } \\
\text { POISSON }\end{array}$ & $\begin{array}{l}\text { ÁNGULO DE } \\
\text { FRICCIÓN } \\
\left.{ }^{\circ}\right)\end{array}$ & $\begin{array}{c}\text { COHESIÓN } \\
\text { (Pa) }\end{array}$ & $\underset{\text { (Pa) }}{\text { TENSIÓN }}$ & \begin{tabular}{|c|} 
PESO \\
UNTTARIO \\
$(\mathrm{Kg} / \mathrm{m} 3)$
\end{tabular} \\
\hline $\mathrm{Lm}$ & 600 & Limolita & $5.37 \mathrm{E} 10$ & 0.26 & 27 & $2.68 \mathrm{E} 7$ & $1.58 \mathrm{E}+06$ & 2720 \\
\hline $\mathrm{Ar}$ & 600 & Arenisca & $2.55 \mathrm{E} 10$ & 0.38 & 28 & $2.7 \mathrm{E} 7$ & $1.17 \mathrm{E}+07$ & 2180 \\
\hline$Q$ & 2000 & Cuarcita & 3.67E10 & 0.18 & 50 & $2.7 \mathrm{E} 7$ & $1.17 \mathrm{E}+07$ & 2640 \\
\hline $\mathrm{Sq}$ & 500 & Esquisto & 3.67E9 & 0.25 & 10 & $7 \mathrm{E} 5$ & $1.17 \mathrm{E}+07$ & 2470 \\
\hline
\end{tabular}

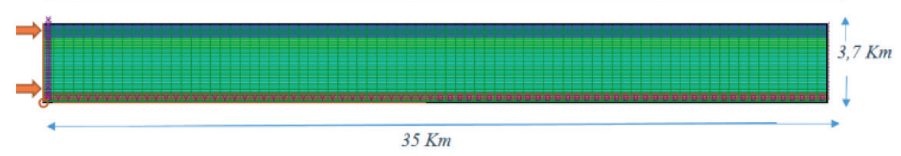

Figura 20: Modelo denudacional

La erosión se modeló considerando la reducción de los parámetros de resistencia del material en los desplazamientos de 2, 4 y $6 \mathrm{~km}$, disminuyendo la cohesión en cada fase en un orden de magnitud de $26.8 \mathrm{MPa}$ a 27 $\mathrm{kPa}$. En la Figura 21 se observa el desarrollo de la zona de falla con respecto al desplazamiento y el incremento de la deformación cortante en las laderas para cada cambio de cohesión, en estas zonas se genera el desprendimiento y depositación del material en su base.

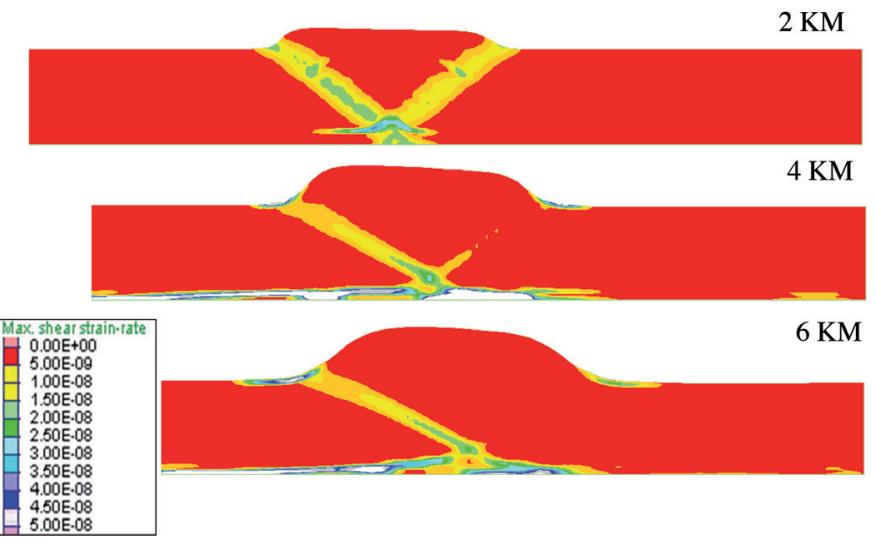

Figura 21: Progresión de la deformación topográfica

Alcanzados $6 \mathrm{~km}$ de desplazamiento, se simula un proceso de descarga por erosión intensiva en dos fases, desprendiendo el material superficial del orden de $50 \mathrm{~m}$ en cada una. En la Figura 22 se muestra la expansión del material por relajación de esfuerzos durante este proceso.

Se analizó la ladera formada en la zona de avance de la cuña de falla, en el cual se realizaron las fases de desprendimiento, depositación y descarga por erosión. Una limitante en la precisión de los resultados fue la cantidad 


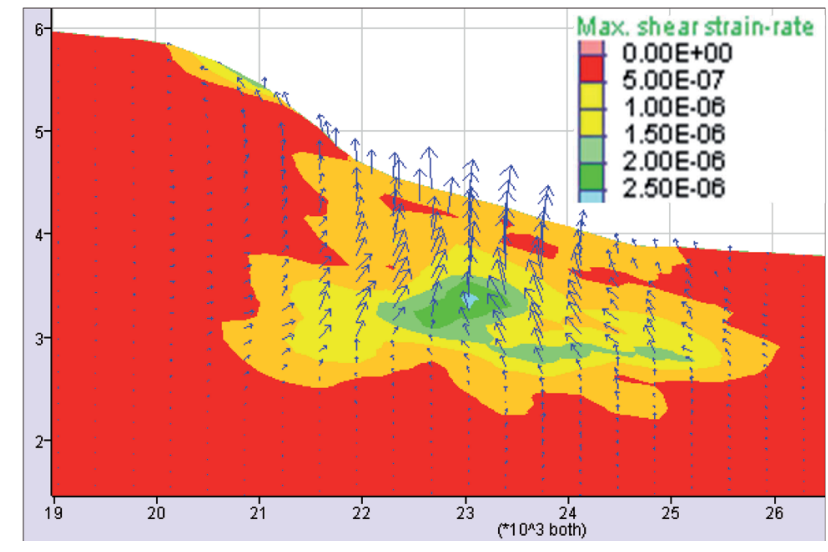

Figura 22: Rebote del material por descarga

de ciclos de re-enmallado utilizados ya que, por la escala del modelo, la interpolación de esfuerzos en cada ciclo pudo presentar errores de convergencia.

El FS obtenido para el escenario morfogenético fue de 1.26 y de 1.29 para el geostático. En la Figura 23 se observan los mecanismos de falla resultantes, los cuales se presentan en la zona superficial de la ladera donde los procesos erosivos actuaron con mayor intensidad. Adicional, se analizaron mecanismos de falla profundos de tipo rotacional, de menor profundidad con respecto a los obtenidos en escenarios de empuje tectónico, siendo más consistentes con los observados en campo. Se obtuvo un FS de 1.26 para el modelo morfogenético y de 1.29 para el convencional.

La magnitud y dirección del esfuerzo principal mayor se presenta en la Figura 24, mostrando una alta semejanza en la magnitud de los esfuerzos del modelo morfogenético en los estratos profundos en comparación con los obtenidos en los escenarios de compresión tectónica. Por otro lado, en las zonas superficiales, especialmente en la mayor parte del cuerpo y corona de la ladera se presenta una dirección de los esfuerzos similar entre los modelos morfogenético y geostático.

\section{Procesos fluviales}

En los ambientes fluviales, la forma del relieve es consecuencia de procesos erosionales y de depositación de ríos actuales o pasados. Estos últimos, pudieron ser de gran magnitud e intensidad produciendo en las laderas diferentes ciclos de carga y de descarga. La descarga se debe a la erosión e incisión del río que desprende y transporta el material. Por otro lado, la carga se debe a a)

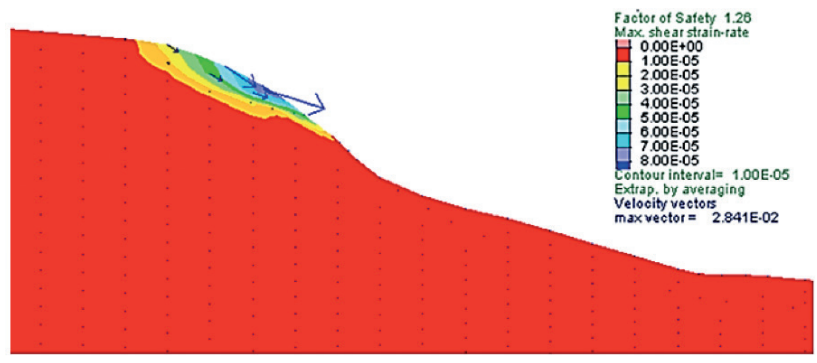

b)

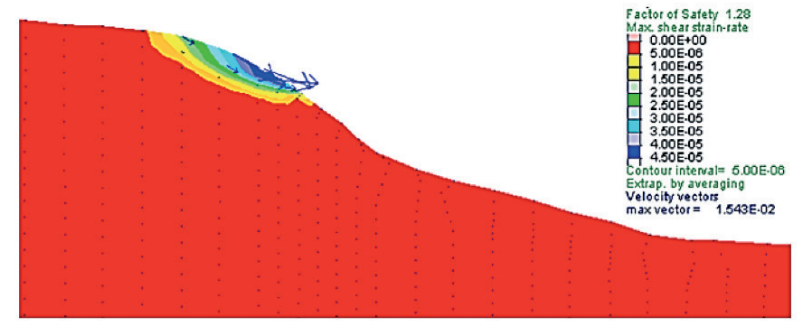

Figura 23: Máxima tasa de deformación cortante en: a) modelo morfogénesis y b) modelo geostático

a)

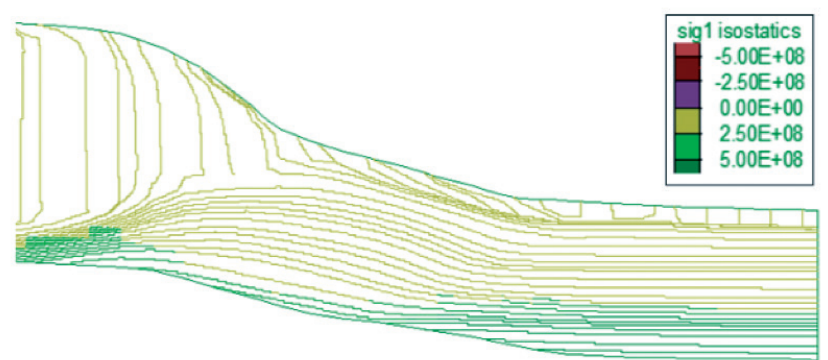

b)

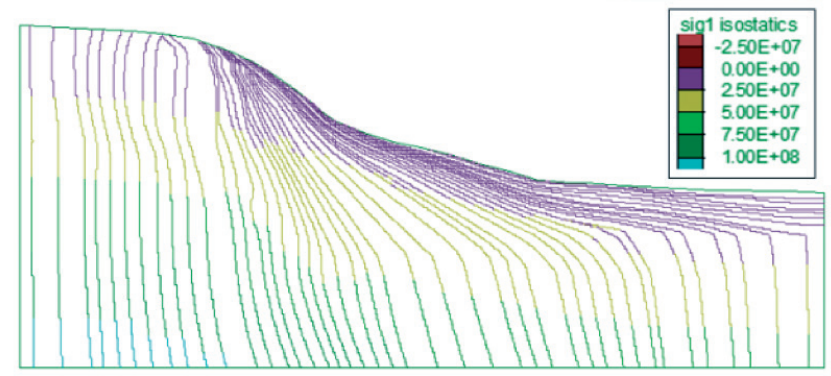

Figura 24: Esfuerzo principal mayor: a) modelo morfogénesis y b) modelo geostático

la depositación del material cuando la fuerza de arrastre del río no es suficiente para seguirlo transportando. Este ambiente es de mayor interés para la ingeniería civil, ya que estas laderas interactúan constantemente con obras de ingeniería y son de una escala espacial relativamente menor a las modeladas previamente. Se realizó el modelo con base a los ciclos de erosión fluvial propuestos por Holmes (1965) para dos ríos cercanos. En la Figura 25 se presenta las dimensiones y propiedades del modelo considerando las diferentes fases de un río desde su juventud hasta su vejez, representadas por las líneas amarillas. 
$\mathrm{y}$, particularmente para el escenario morfogenético, al desconfinamiento por procesos de erosión fluvial. Adicional, se observa que los vectores de velocidad son de mayor magnitud para este escenario. a)

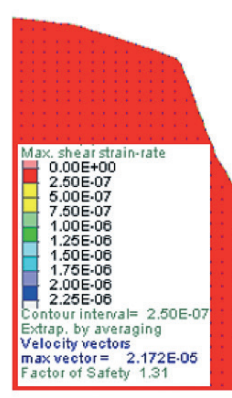

b)

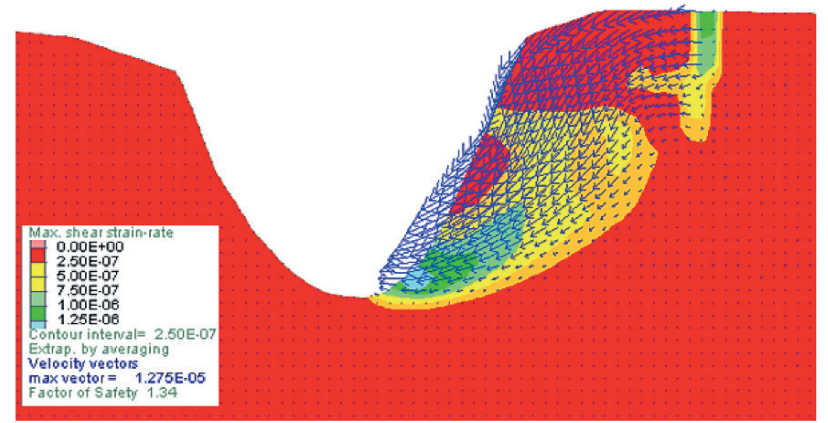

Figura 29: Máxima tasa de deformación cortante en: a) modelo morfogénesis y b) modelo geoestático

\section{Modelo de fases progresivas}

La forma del relieve actual es consecuencia de varios procesos geomorfológicos y se requiere un amplio análisis y una correcta interpretación de las evidencias en campo para identificar cuales actuaron sobre la ladera de estudio. Las modelaciones presentadas previamente se realizaron considerando el proceso geomorfológico predominante para cada ambiente, pero generalmente, los diferentes procesos actúan de manera simultánea o consecutiva en distintas intensidades. Para conocer la influencia de esta secuencia de procesos se realiza un modelo de fases progresivas, en el cual, se inicia el levantamiento del relieve por procesos tectónicos compresionales y posteriormente se modelan procesos denudacionales (erosivos y fluviales). En la Figura 30 se presentan las dimensiones y los parámetros del modelo. Se propuso la intercalación de areniscas con shales de acuerdo a las modelaciones realizadas por Ruh et al. (2012), con el objetivo de observar el plegamiento durante la compresión tectónica.

\begin{tabular}{|c|c|c|c|c|c|c|c|}
\hline ID & $\begin{array}{c}\text { ESPESOR } \\
(\mathbf{m})\end{array}$ & NOMBRE & $\begin{array}{c}\text { MODULO DE } \\
\text { ELASTICIDAD } \\
(\mathbf{P a})\end{array}$ & $\begin{array}{c}\text { RELACIÓN } \\
\text { DE } \\
\text { POISSON }\end{array}$ & $\begin{array}{c}\text { ÁNGULO DE } \\
\text { FRICCION } \\
\left({ }^{\circ}\right.\end{array}$ & $\begin{array}{c}\text { COHESIÓN } \\
(\text { Pa) }\end{array}$ & $\begin{array}{c}\text { PESO } \\
\text { UNITARIO } \\
(\mathbf{K g} / \mathbf{m} 3)\end{array}$ \\
\hline $\mathrm{Ar}$ & 3,00 & Arenisca 1 & $2.54 \mathrm{E} 10$ & 0.38 & 30 & $2.7 \mathrm{E}+07$ & 2100 \\
\hline $\mathrm{Sh}$ & 0.5 & Shale 1 & $5.37 \mathrm{E} 10$ & 0.25 & 10 & $7 \mathrm{E} 5$ & 2720 \\
\hline $\mathrm{Ar} 2$ & 1,00 & Arenisca 2 & $2.54 \mathrm{E} 10$ & 0.38 & 30 & $2.72 \mathrm{E}+07$ & 2100 \\
\hline $\mathrm{Sh} 2$ & 0.5 & Shale & $5.37 \mathrm{E} 10$ & 0.25 & 10 & $7 \mathrm{E} 5$ & 2720 \\
\hline
\end{tabular}

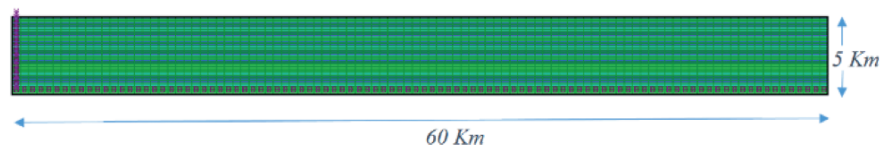

Figura 30: Modelo de fases progresivas

\section{Fase 1. Compresión tectónica}

La primera fase consiste en la compresión tectónica. A diferencia de las modelaciones previamente analizadas, en este caso se continuó con los desplazamientos laterales hasta generar una segunda cuña de falla y así simular un canal natural entre las dos colinas resultantes, para posteriormente modelar procesos fluviales. En la Figura 31 se presenta los resultados del plegamiento para un desplazamiento de $5 \mathrm{~km}$ y para uno de $9.4 \mathrm{~km}$.

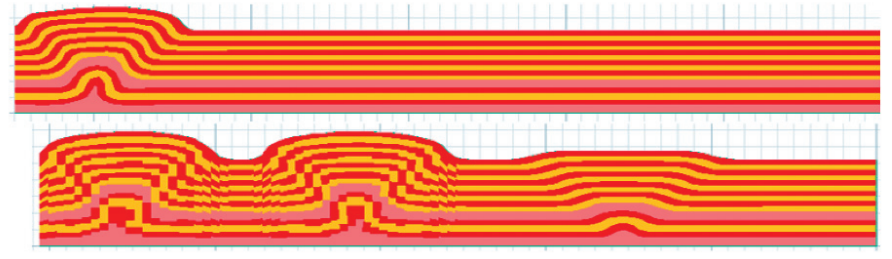

Figura 31: Levantamiento topográfico durante la fase de empuje tectónico

\section{Fase 2. Denudacional-erosión}

A partir de la geoforma resultante de la primera fase, se modelan los procesos erosivos en las laderas del canal natural entre las dos montañas formadas. Siguiendo la metodología del modelo de erosión se simula la meteorización del material gradualmente en 5 fases, alcanzando un espesor superficial de $100 \mathrm{~m}$. Se observa que a medida que se degrada el material este se desprende y deposita en la base de la ladera, suavizando el relieve y disminuyendo su pendiente.

\section{Fase 3. Fluvial}

En la tercera fase se simula la erosión fluvial realizando una descarga de un espesor de $50 \mathrm{~m}$ del material en el lecho del cauce. En la Figura 32 se presenta la morfometría final del modelo, donde se observan escarpes de afloramiento de 
roca y en su base un material producto de la depositación. El FS obtenido para la fase final fue de 1.42, menor al obtenido para un escenario geostático (1.58). En la Figura 33 se presenta el mecanismo de falla para ambos escenarios el cual es de tipo traslacional o de flujo de detritos.

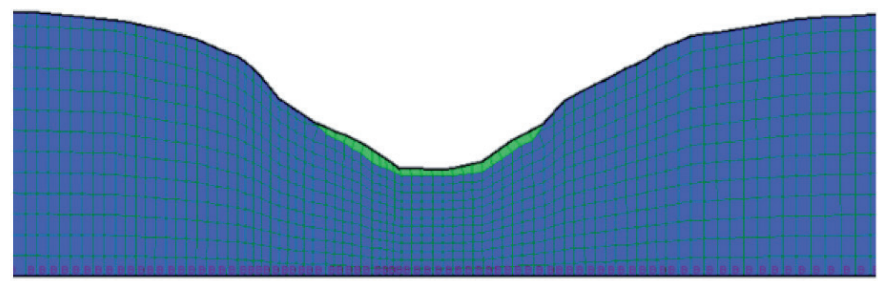

Figura 32: Morfometría fase final del modelo progresivo

a)

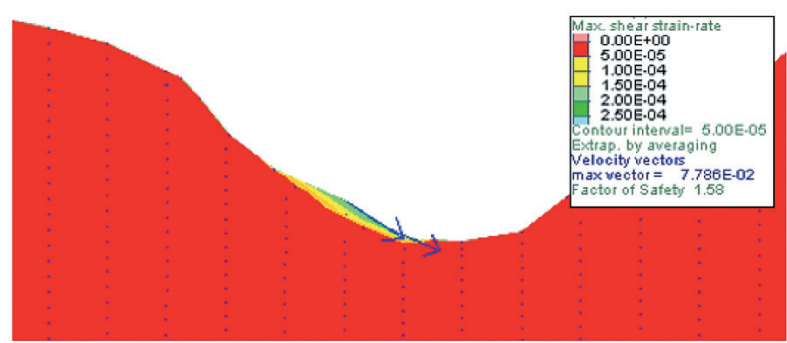

b)

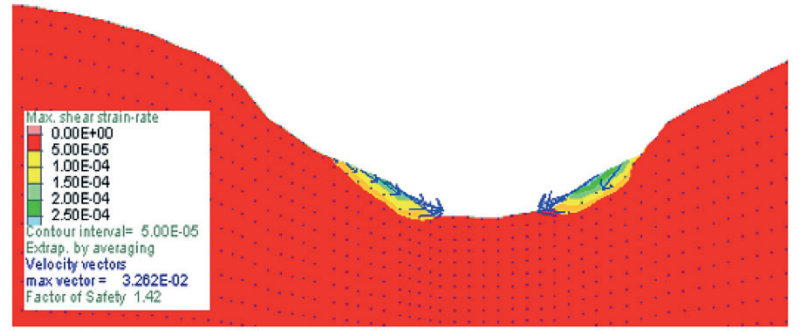

Figura 33: Máxima tasa de deformación cortante en: a) modelo morfogénesis y b) modelo geoestático

En la Figura 34 se presenta la condición del esfuerzo principal mayor en la ladera para ambos escenarios, se observa que las magnitudes en el escenario morfogenético es de 2 hasta 10 veces mayor para los estratos profundos y presenta una orientación horizontal en comparación con el escenario convencional. Esta condición se ha presentado en todas las modelaciones de procesos de empuje tectónico. Por otro lado, para las zonas superficiales de la corona de la ladera las magnitudes son más similares. Los esfuerzos resultantes indican que los procesos tectónicos tienen mayor influencia a grandes profundidades que superficialmente, en cambio, en la zona superficial se observa mayor similitud en cuanto a dirección y magnitud de los esfuerzos entre ambos escenarios, siendo los procesos de erosión los que tiene mayor influencia. Adicional, la zona de falla en
el escenario morfogenético influye en la rotación de los
esfuerzos principales.
a)
b)
a)
FLAC para el desarrollo de los modelos planteados, son
adecuadas y permiten obtener resultados para grandes
deformaciones, programar funciones de re-enmallado y
calcular el factor de seguridad FS.
figura 34 : Esfuerzo principal mayor: a) modelo morfogénesis y
b) modelo geostático
Conclusiones
Al realizar el análisis cuantitativo de los factores
gemorfológicos, particularmente de la morfogénesis, se
determinó que los procesos de formación geomorfológica
tienen influencia en la condición de esfuerzos de las laderas
y por consiguiente en su estabilidad. El grado de influencia
depende del ambiente de formación, la escala de análisis
estudiada y el tipo de intervención a realizar. De manera
general, los procesos superficiales de erosión disminuyen
el esfuerzo principal menor generando mayores zonas de
tensión, mientras que los procesos de empuje tectónico

que tiene mayor influencia. Adicional, la zona de falla en
el escenario morfogenético influye en la rotación de los
esfuerzos principales.
a)
b)
F)
adecuadas y permiten obtener resultados para grandes
deformaciones, programar funciones de re-enmallado y
calcular el factor de seguridad FS.
Figura 34 : Esfuerzo principal mayor: a) modelo morfogénesis y
b) modelo geostático
Conclusiones
Al realizar el análisis cuantitativo de los factores
gemorfológicos, particularmente de la morfogénesis, se
determinó que los procesos de formación geomorfológica
tienen influencia en la condición de esfuerzos de las laderas
y por consiguiente en su estabilidad. El grado de influencia
depende del ambiente de formación, la escala de análisis
estudiada y el tipo de intervención a realizar. De manera
general, los procesos superficiales de erosión disminuyen
el esfuerzo principal menor generando mayores zonas de
tensión, mientras que los procesos de empuje tectónico
anen alta influencia en profundidad, importante al

que tiene mayor influencia. Adicional, la zona de falla en
el escenario morfogenético influye en la rotación de los
esfuerzos principales.
a)
b)
F)
adecuadas y permiten obtener resultados para grandes
deformaciones, programar funciones de re-enmallado y
calcular el factor de seguridad FS.
Figura 34 : Esfuerzo principal mayor: a) modelo morfogénesis y
b) modelo geostático
Conclusiones
Al realizar el análisis cuantitativo de los factores
gemorfológicos, particularmente de la morfogénesis, se
determinó que los procesos de formación geomorfológica
tienen influencia en la condición de esfuerzos de las laderas
y por consiguiente en su estabilidad. El grado de influencia
depende del ambiente de formación, la escala de análisis
estudiada y el tipo de intervención a realizar. De manera
general, los procesos superficiales de erosión disminuyen
el esfuerzo principal menor generando mayores zonas de
tensión, mientras que los procesos de empuje tectónico
anen alta influencia en profundidad, importante al

a)

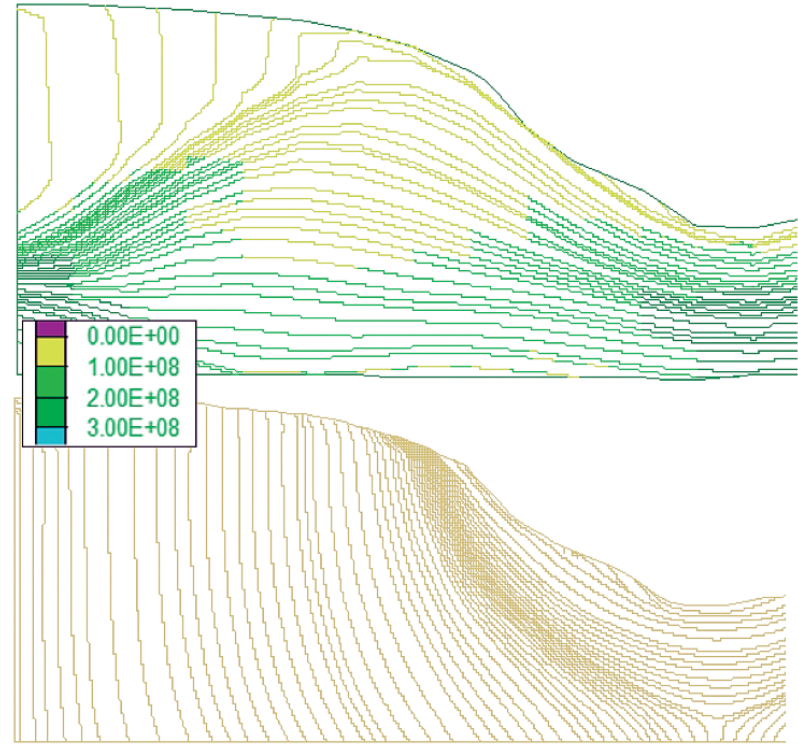

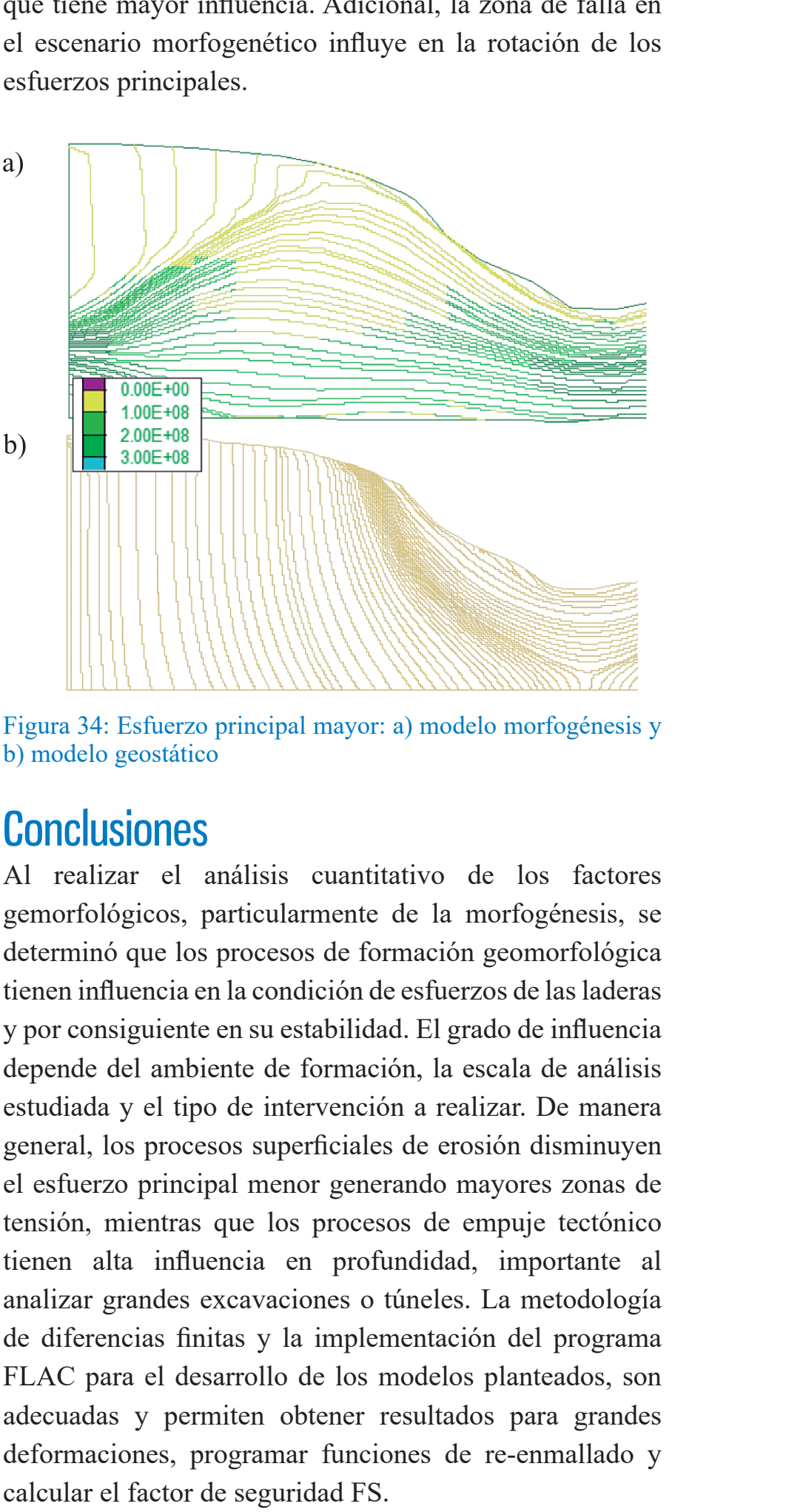

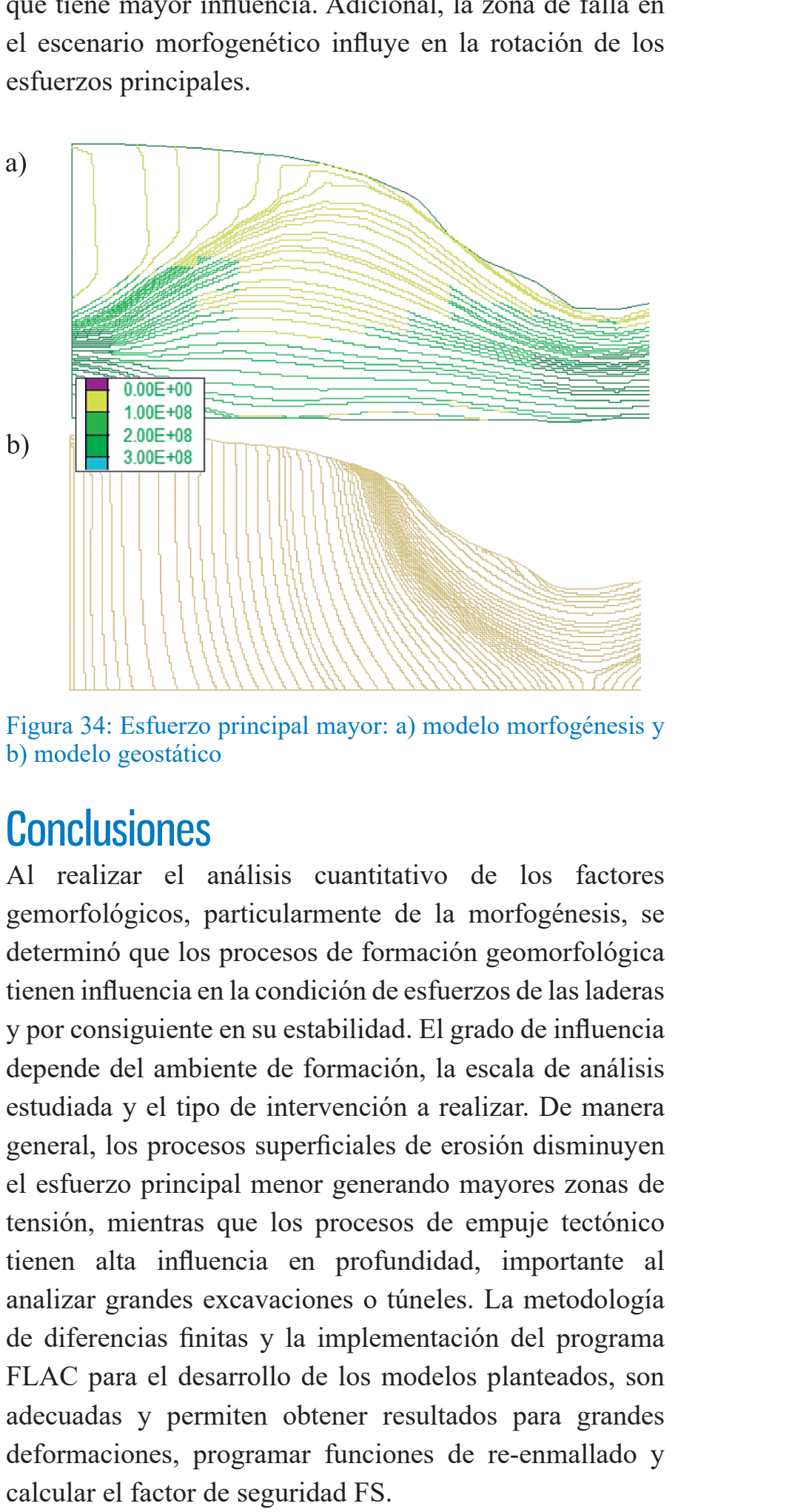

que tiene mayor influencia. Adicional, la zona de falla en
el escenario morfogenético influye en la rotación de los
esfuerzos principales.
a)
b)
Figura 34: Esfuerzo principal mayor: a) modelo morfogénesis y
b) modelo geostático
Conclusiones
Al realizar el análisis cuantitativo de los factores
gemorfológicos, particularmente de la morfogénesis, se
determinó que los procesos de formación geomorfológica
tienen influencia en la condición de esfuerzos de las laderas
y por consiguiente en su estabilidad. El grado de influencia
depende del ambiente de formación, la escala de ańlisis
estudiada y el tipo de intervención a realizar. De manera
general, los procesos superficiales de erosión disminuyen
el esfuerzo principal menor generando mayores zonas de
tensión, mientras que los procesos de empuje tectónico
tienen alta influencia en profundidad, importante al
analizar grandes excavaciones o túneles. La metodología
de diferencias finitas y la implementación del programa
FLAC para el desarrollo de los modelos planteados, son
adecuadas y permiten obtener resultados para grandes
deformaciones, programar funciones de re-enmallado y
calcular el factor de seguridad Fs.

que tiene mayor influencia. Adicional, la zona de falla en
el escenario morfogenético influye en la rotación de los
esfuerzos principales.
a)
b)
F)
adecuadas y permiten obtener resultados para grandes
deformaciones, programar funciones de re-enmallado y
calcular el factor de seguridad FS.
Figura 34 : Esfuerzo principal mayor: a) modelo morfogénesis y
b) modelo geostático
Conclusiones
Al realizar el análisis cuantitativo de los factores
gemorfológicos, particularmente de la morfogénesis, se
determinó que los procesos de formación geomorfológica
tienen influencia en la condición de esfuerzos de las laderas
y por consiguiente en su estabilidad. El grado de influencia
depende del ambiente de formación, la escala de análisis
estudiada y el tipo de intervención a realizar. De manera
general, los procesos superficiales de erosión disminuyen
el esfuerzo principal menor generando mayores zonas de
tensión, mientras que los procesos de empuje tectónico
anen alta influencia en profundidad, importante al

que tiene mayor influencia. Adicional, la zona de falla en
el escenario morfogenético influye en la rotación de los
esfuerzos principales.
a)
b)
F)
adecuadas y permiten obtener resultados para grandes
deformaciones, programar funciones de re-enmallado y
calcular el factor de seguridad FS.
Figura 34 : Esfuerzo principal mayor: a) modelo morfogénesis y
b) modelo geostático
Conclusiones
Al realizar el análisis cuantitativo de los factores
gemorfológicos, particularmente de la morfogénesis, se
determinó que los procesos de formación geomorfológica
tienen influencia en la condición de esfuerzos de las laderas
y por consiguiente en su estabilidad. El grado de influencia
depende del ambiente de formación, la escala de análisis
estudiada y el tipo de intervención a realizar. De manera
general, los procesos superficiales de erosión disminuyen
el esfuerzo principal menor generando mayores zonas de
tensión, mientras que los procesos de empuje tectónico
anen alta influencia en profundidad, importante al

que tiene mayor influencia. Adicional, la zona de falla en
el escenario morfogenético influye en la rotación de los
esfuerzos principales.
a)
b)
F)
adecuadas y permiten obtener resultados para grandes
deformaciones, programar funciones de re-enmallado y
calcular el factor de seguridad FS.
Figura 34 : Esfuerzo principal mayor: a) modelo morfogénesis y
b) modelo geostático
Conclusiones
Al realizar el análisis cuantitativo de los factores
gemorfológicos, particularmente de la morfogénesis, se
determinó que los procesos de formación geomorfológica
tienen influencia en la condición de esfuerzos de las laderas
y por consiguiente en su estabilidad. El grado de influencia
depende del ambiente de formación, la escala de análisis
estudiada y el tipo de intervención a realizar. De manera
general, los procesos superficiales de erosión disminuyen
el esfuerzo principal menor generando mayores zonas de
tensión, mientras que los procesos de empuje tectónico
anen alta influencia en profundidad, importante al

que tiene mayor influencia. Adicional, la zona de falla en
el escenario morfogenético influye en la rotación de los
esfuerzos principales.
a)
b)
F)
adecuadas y permiten obtener resultados para grandes
deformaciones, programar funciones de re-enmallado y
calcular el factor de seguridad FS.
Figura 34 : Esfuerzo principal mayor: a) modelo morfogénesis y
b) modelo geostático
Conclusiones
Al realizar el análisis cuantitativo de los factores
gemorfológicos, particularmente de la morfogénesis, se
determinó que los procesos de formación geomorfológica
tienen influencia en la condición de esfuerzos de las laderas
y por consiguiente en su estabilidad. El grado de influencia
depende del ambiente de formación, la escala de análisis
estudiada y el tipo de intervención a realizar. De manera
general, los procesos superficiales de erosión disminuyen
el esfuerzo principal menor generando mayores zonas de
tensión, mientras que los procesos de empuje tectónico
anen alta influencia en profundidad, importante al

que tiene mayor influencia. Adicional, la zona de falla en
el escenario morfogenético influye en la rotación de los
esfuerzos principales.
a)
b)
F)
adecuadas y permiten obtener resultados para grandes
deformaciones, programar funciones de re-enmallado y
calcular el factor de seguridad FS.
Figura 34 : Esfuerzo principal mayor: a) modelo morfogénesis y
b) modelo geostático
Conclusiones
Al realizar el análisis cuantitativo de los factores
gemorfológicos, particularmente de la morfogénesis, se
determinó que los procesos de formación geomorfológica
tienen influencia en la condición de esfuerzos de las laderas
y por consiguiente en su estabilidad. El grado de influencia
depende del ambiente de formación, la escala de análisis
estudiada y el tipo de intervención a realizar. De manera
general, los procesos superficiales de erosión disminuyen
el esfuerzo principal menor generando mayores zonas de
tensión, mientras que los procesos de empuje tectónico
anen alta influencia en profundidad, importante al

que tiene mayor influencia. Adicional, la zona de falla en
el escenario morfogenético influye en la rotación de los
esfuerzos principales.
a)
b)
F)
adecuadas y permiten obtener resultados para grandes
deformaciones, programar funciones de re-enmallado y
calcular el factor de seguridad FS.
Figura 34 : Esfuerzo principal mayor: a) modelo morfogénesis y
b) modelo geostático
Conclusiones
Al realizar el análisis cuantitativo de los factores
gemorfológicos, particularmente de la morfogénesis, se
determinó que los procesos de formación geomorfológica
tienen influencia en la condición de esfuerzos de las laderas
y por consiguiente en su estabilidad. El grado de influencia
depende del ambiente de formación, la escala de análisis
estudiada y el tipo de intervención a realizar. De manera
general, los procesos superficiales de erosión disminuyen
el esfuerzo principal menor generando mayores zonas de
tensión, mientras que los procesos de empuje tectónico
anen alta influencia en profundidad, importante al

que tiene mayor influencia. Adicional, la zona de falla en
el escenario morfogenético influye en la rotación de los
esfuerzos principales.
a)
b)
F)
adecuadas y permiten obtener resultados para grandes
deformaciones, programar funciones de re-enmallado y
calcular el factor de seguridad FS.
Figura 34 : Esfuerzo principal mayor: a) modelo morfogénesis y
b) modelo geostático
Conclusiones
Al realizar el análisis cuantitativo de los factores
gemorfológicos, particularmente de la morfogénesis, se
determinó que los procesos de formación geomorfológica
tienen influencia en la condición de esfuerzos de las laderas
y por consiguiente en su estabilidad. El grado de influencia
depende del ambiente de formación, la escala de análisis
estudiada y el tipo de intervención a realizar. De manera
general, los procesos superficiales de erosión disminuyen
el esfuerzo principal menor generando mayores zonas de
tensión, mientras que los procesos de empuje tectónico
anen alta influencia en profundidad, importante al

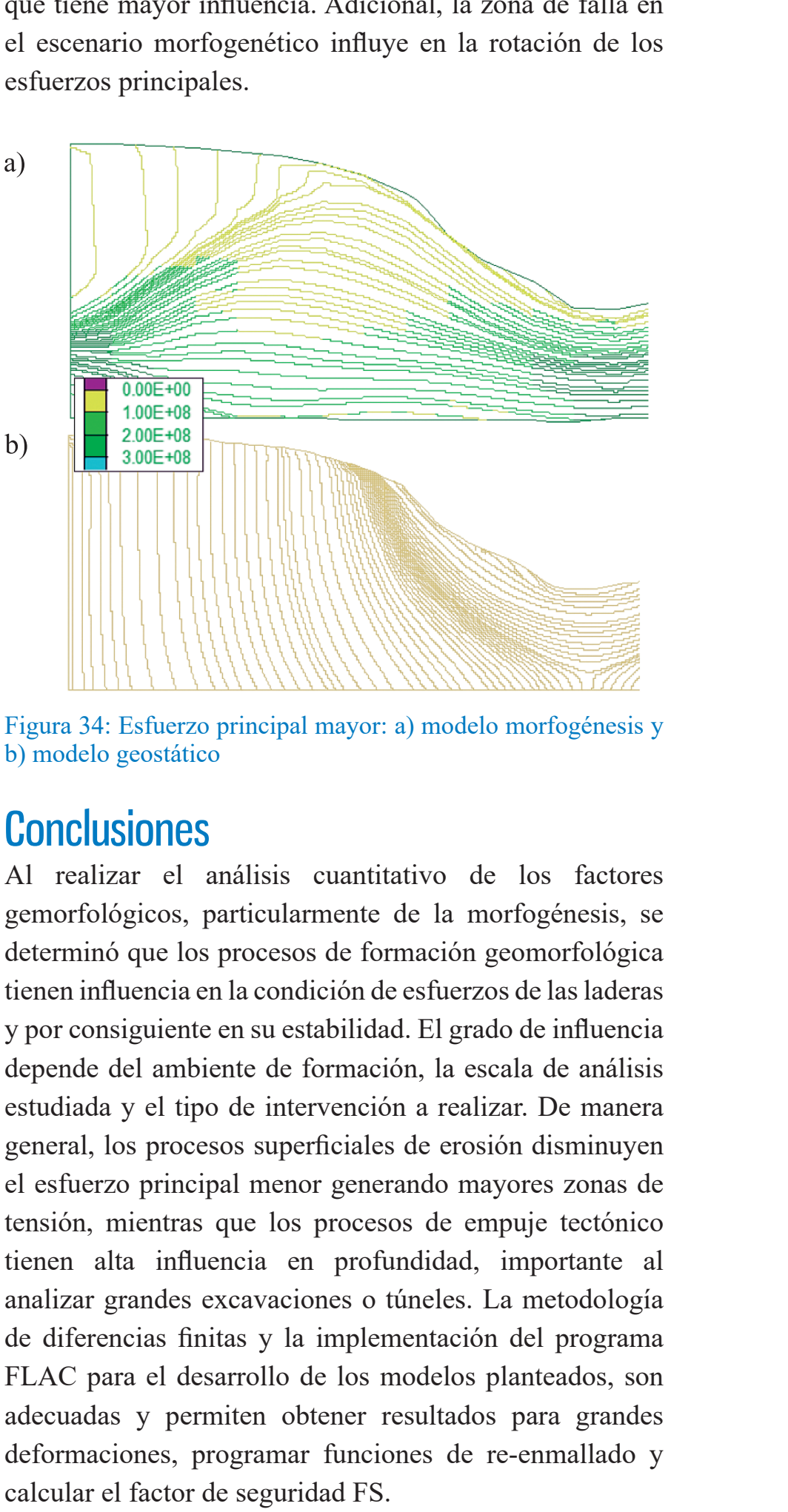

que tiene mayor influencia. Adicional, la zona de falla en
el escenario morfogenético influye en la rotación de los
esfuerzos principales.
a)
b)
F)
adecuadas y permiten obtener resultados para grandes
deformaciones, programar funciones de re-enmallado y
calcular el factor de seguridad FS.
Figura 34 : Esfuerzo principal mayor: a) modelo morfogénesis y
b) modelo geostático
Conclusiones
Al realizar el análisis cuantitativo de los factores
gemorfológicos, particularmente de la morfogénesis, se
determinó que los procesos de formación geomorfológica
tienen influencia en la condición de esfuerzos de las laderas
y por consiguiente en su estabilidad. El grado de influencia
depende del ambiente de formación, la escala de análisis
estudiada y el tipo de intervención a realizar. De manera
general, los procesos superficiales de erosión disminuyen
el esfuerzo principal menor generando mayores zonas de
tensión, mientras que los procesos de empuje tectónico
anen alta influencia en profundidad, importante al

que tiene mayor influencia. Adicional, la zona de falla en
el escenario morfogenético influye en la rotación de los
esfuerzos principales.
a)
b)
F)
adecuadas y permiten obtener resultados para grandes
deformaciones, programar funciones de re-enmallado y
calcular el factor de seguridad FS.
Figura 34 : Esfuerzo principal mayor: a) modelo morfogénesis y
b) modelo geostático
Conclusiones
Al realizar el análisis cuantitativo de los factores
gemorfológicos, particularmente de la morfogénesis, se
determinó que los procesos de formación geomorfológica
tienen influencia en la condición de esfuerzos de las laderas
y por consiguiente en su estabilidad. El grado de influencia
depende del ambiente de formación, la escala de análisis
estudiada y el tipo de intervención a realizar. De manera
general, los procesos superficiales de erosión disminuyen
el esfuerzo principal menor generando mayores zonas de
tensión, mientras que los procesos de empuje tectónico
anen alta influencia en profundidad, importante al

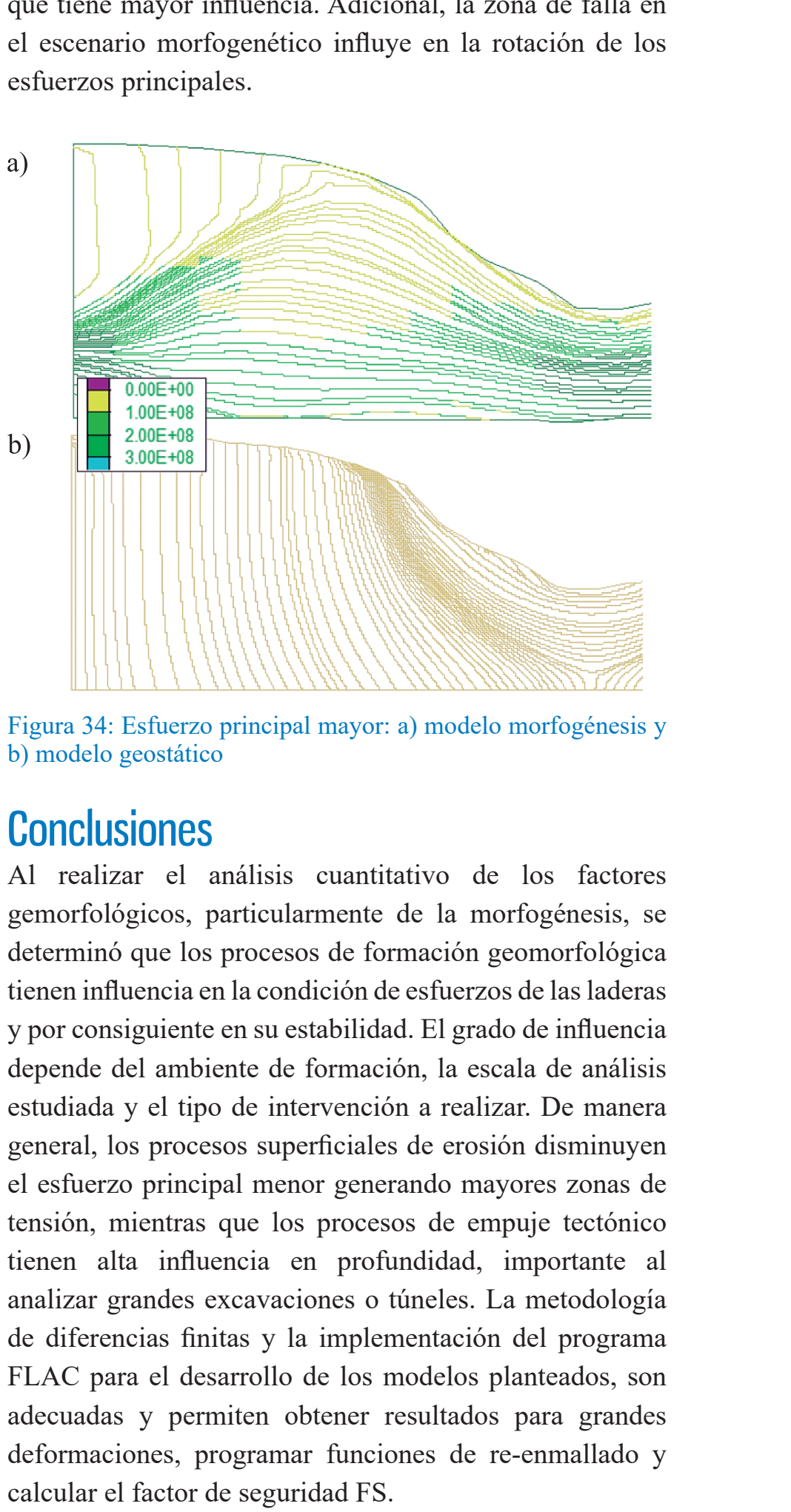

que tiene mayor influencia. Adicional, la zona de falla en
el escenario morfogenético influye en la rotación de los
esfuerzos principales.
a)
b)
F)
adecuadas y permiten obtener resultados para grandes
deformaciones, programar funciones de re-enmallado y
calcular el factor de seguridad FS.
Figura 34 : Esfuerzo principal mayor: a) modelo morfogénesis y
b) modelo geostático
Conclusiones
Al realizar el análisis cuantitativo de los factores
gemorfológicos, particularmente de la morfogénesis, se
determinó que los procesos de formación geomorfológica
tienen influencia en la condición de esfuerzos de las laderas
y por consiguiente en su estabilidad. El grado de influencia
depende del ambiente de formación, la escala de análisis
estudiada y el tipo de intervención a realizar. De manera
general, los procesos superficiales de erosión disminuyen
el esfuerzo principal menor generando mayores zonas de
tensión, mientras que los procesos de empuje tectónico
anen alta influencia en profundidad, importante al

que tiene mayor influencia. Adicional, la zona de falla en
el escenario morfogenético influye en la rotación de los
esfuerzos principales.
a)
b)
F)
adecuadas y permiten obtener resultados para grandes
deformaciones, programar funciones de re-enmallado y
calcular el factor de seguridad FS.
Figura 34 : Esfuerzo principal mayor: a) modelo morfogénesis y
b) modelo geostático
Conclusiones
Al realizar el análisis cuantitativo de los factores
gemorfológicos, particularmente de la morfogénesis, se
determinó que los procesos de formación geomorfológica
tienen influencia en la condición de esfuerzos de las laderas
y por consiguiente en su estabilidad. El grado de influencia
depende del ambiente de formación, la escala de análisis
estudiada y el tipo de intervención a realizar. De manera
general, los procesos superficiales de erosión disminuyen
el esfuerzo principal menor generando mayores zonas de
tensión, mientras que los procesos de empuje tectónico
anen alta influencia en profundidad, importante al

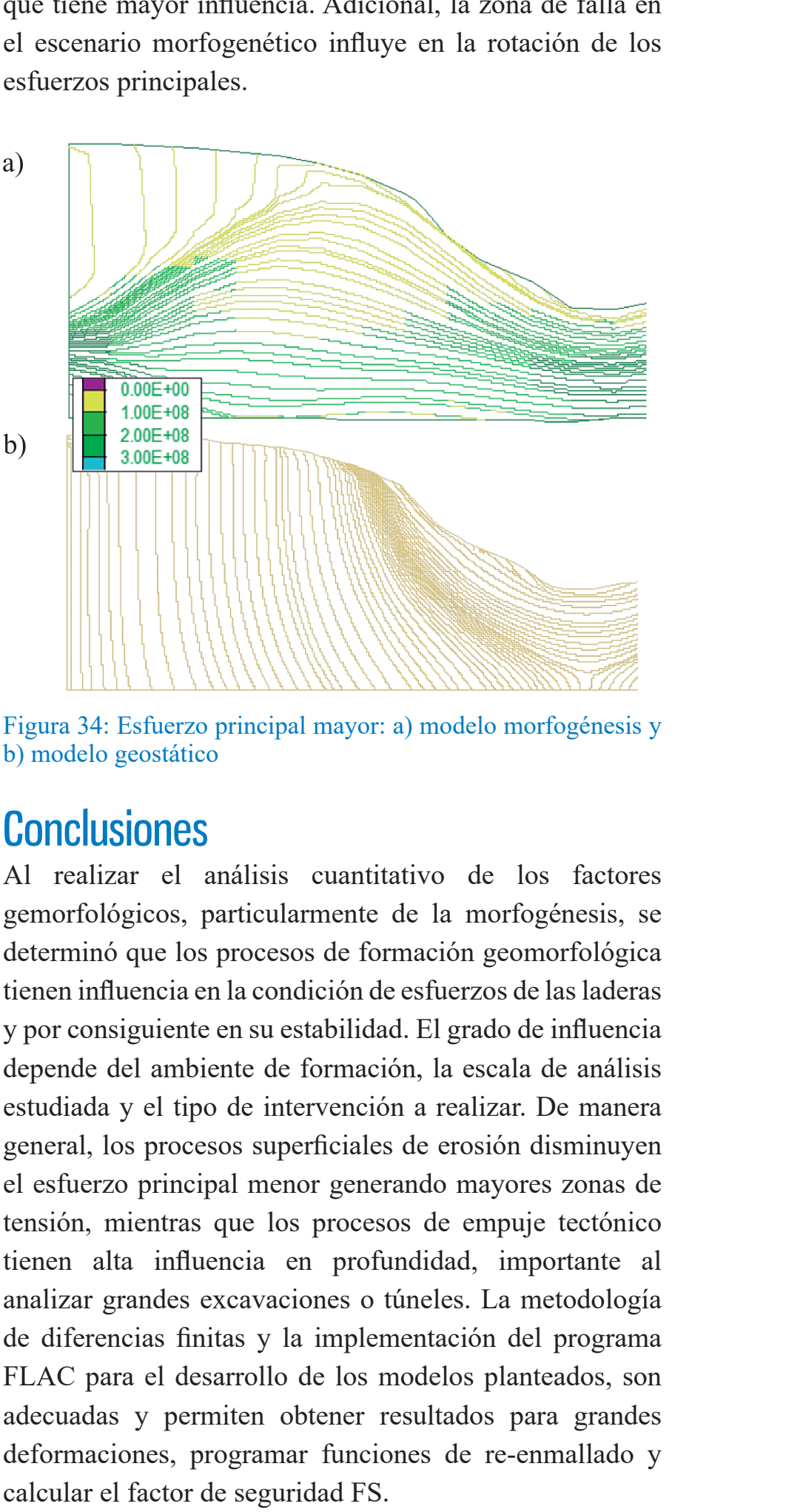

que tiene mayor influencia. Adicional, la zona de falla en
el escenario morfogenético influye en la rotación de los
esfuerzos principales.
a)
b)
F)
adecuadas y permiten obtener resultados para grandes
deformaciones, programar funciones de re-enmallado y
calcular el factor de seguridad FS.
Figura 34 : Esfuerzo principal mayor: a) modelo morfogénesis y
b) modelo geostático
Conclusiones
Al realizar el análisis cuantitativo de los factores
gemorfológicos, particularmente de la morfogénesis, se
determinó que los procesos de formación geomorfológica
tienen influencia en la condición de esfuerzos de las laderas
y por consiguiente en su estabilidad. El grado de influencia
depende del ambiente de formación, la escala de análisis
estudiada y el tipo de intervención a realizar. De manera
general, los procesos superficiales de erosión disminuyen
el esfuerzo principal menor generando mayores zonas de
tensión, mientras que los procesos de empuje tectónico
anen alta influencia en profundidad, importante al

que tiene mayor influencia. Adicional, la zona de falla en
el escenario morfogenético influye en la rotación de los
esfuerzos principales.
a)
b)
F)
adecuadas y permiten obtener resultados para grandes
deformaciones, programar funciones de re-enmallado y
calcular el factor de seguridad FS.
Figura 34 : Esfuerzo principal mayor: a) modelo morfogénesis y
b) modelo geostático
Conclusiones
Al realizar el análisis cuantitativo de los factores
gemorfológicos, particularmente de la morfogénesis, se
determinó que los procesos de formación geomorfológica
tienen influencia en la condición de esfuerzos de las laderas
y por consiguiente en su estabilidad. El grado de influencia
depende del ambiente de formación, la escala de análisis
estudiada y el tipo de intervención a realizar. De manera
general, los procesos superficiales de erosión disminuyen
el esfuerzo principal menor generando mayores zonas de
tensión, mientras que los procesos de empuje tectónico
anen alta influencia en profundidad, importante al

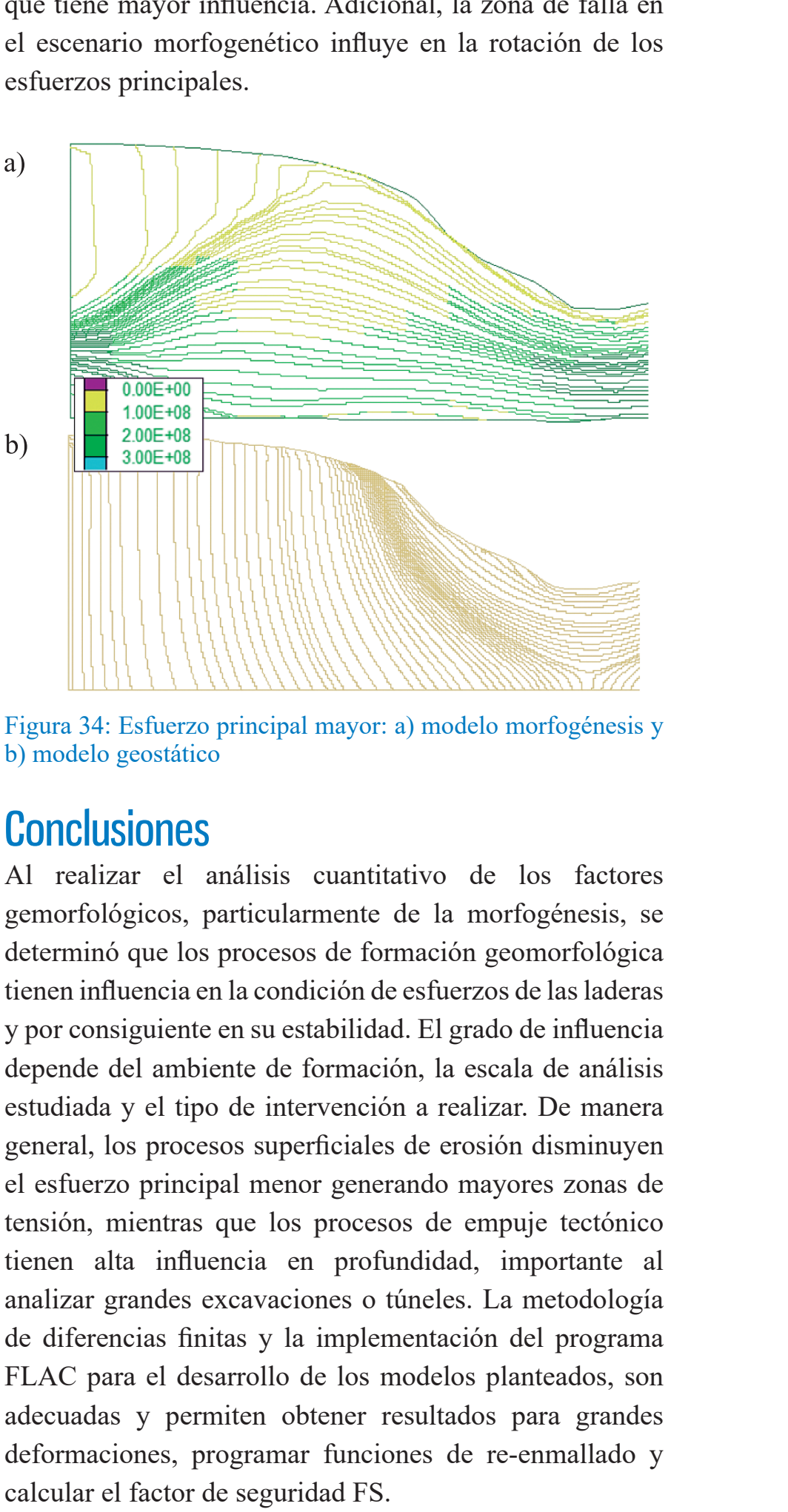


fina e incluya modelos reológicos más avanzados. No se recomienda seguir enfocando las modelaciones de estos escenarios para analizar esta influencia por la complejidad de los análisis y la baja aplicabilidad para el área de la Ingeniería Civil.

Los modelos de ambientes estructurales desarrollaron zonas de falla de concentración de deformaciones cortantes, las cuales se extienden hasta la superficie y afloran en la base de las laderas. Estas zonas de falla influencian la condición de esfuerzos en la ladera, y en consecuencia, su FS y mecanismos de falla. Los escenarios de empuje tectónico muestran una orientación del esfuerzo principal mayor en sentido horizontal, diferente a una condición geostática convencional, donde el esfuerzo principal tiende a ser vertical. La diferencia encontrada entre las magnitudes de los esfuerzos y los FS indican una alta influencia de los procesos de empuje tectónico, que para obras de gran magnitud en estos ambientes se debería analizar a detalle su influencia.

La modelación de los ambientes de erosión indican que la descarga del material superficial influye en la orientación y magnitud de los esfuerzos, ya que el desconfinamiento disminuye los esfuerzos de compresión tectónica, obteniendo una distribución de esfuerzos más similar a una condición geostática. Sin embargo, los resultados indican que el FS sigue siendo menor al considerar la morfogénesis. Los modelos que mostraron morfometrías más similares a las observadas en la corteza terrestre fueron aquellos que incluían procesos de erosión. Para los modelos en que sólo se consideró el empuje tectónico se obtuvieron laderas de pendientes bajas, de largas longitudes y con FS muy altos.

La modelación del escenario fluvial mostró una alta influencia de los procesos de erosión fluvial en la estabilidad. La descarga del material erosionado genera el desconfinamiento de la ladera, produciendo esfuerzos de tensión tanto en su corona como en su base, dando como resultado mecanismos de falla compuestos (grieta de tracción en la corona y una falla rotacional a mayor profundidad). Se concluye que es necesario considerar estos esfuerzos de tensión cuando se intervengan estas laderas con obras de corte o de terraceo. A pesar de que los modelos que presentaron resultados de mayor diferencia con el convencional fueron los del ambiente estructural, se concluyó que el FS, calculado a partir de un criterio de falla tipo Mohr - Coulomb, no es un criterio adecuado de comparación en estos ambientes ya que se generaron mecanismos de falla no consistentes con los evidenciados en campo. Para una mejor comparación, se deberán utilizar modelos constitutivos más complejos, que consideren la influencia de las diaclasas en los macizos, o utilizar otra metodología de modelación numérica.

\section{Referencias}

Abbasi, I.A., Hersi, O.S. and Al-Harthy, A. (2014). Late Cretaceous Conglomerates of the Qahlah Formation, north Oman. In Tectonic Evolution of the Oman Mountains, Rollinson, Searle, Abbasi, Al-Lazki and Al-Kindi (eds.). Geological Society London, UK, Special Publications 392, 325-341

Barnichon, J.D. (1998). Finite element modelling in structural and petroleum geology. $\mathrm{PhD}$ thesis, University of Liege, Belgium Buiter, S.J.H., Yu, A., Babeyko, S.E., Gerya, T.V., Kaus, B.J.P., Kellner, A., Schreurs, G. and Yamada, Y. (2006). The numerical sandbox: comparison of model results for a shortening and an extension experiment. In Analogue and Numerical Modelling of Crustal-Scale Processes, Buiter and Scheurs (eds.), Geological Society of London, UK, Special Publication 253, 29-64

Colletta, B., Letouzey, J., Pinedo, R., Ballard, J.F. and Balé, P. (1991). Computerized X-ray tomography analysis of sandbox models: examples of thin-skinned thrust systems. Geology 19(11), 1063-1067

Ellis, S., Schreurs, G. and Panien, M. (2004). Comparisons between analogue and numerical models of thrust wedge development. Journal of Structural Geology 26(9), 1659 -1675

FLAC 2D (2011). FLAC - Fast Lagrangian Analysis of Continua Dynamic Analysis. Itasca Consulting Group Inc., Minneapolis, Minnesota, USA

Ford, J.T. (2015). Computer models of a basement involved fault propagation fold during the Laramide Orogeny around Las Vegas, New Mexico. MSc thesis, The University of Texas at Arlington, USA

Han, Y., Cundall, P.A. and Hart, R.D. (2008). Automatic remeshing logic in large strain continuum simulations. In Continuum and Distinct Element Numerical Modeling in GeoEngineering. Hart, Detournay and Cundall (eds.), Itasca Consulting Group Inc. Minneapolis, USA, paper 02-02 
Henk, A. and Nemcok, M. (2008). Stress and fracture prediction in inverted half-graben structures. Journal of Structural Geology 30(1), $81-97$

Holmes, A. (1965). Principles of physical geology. Nelson, London, UK

Hutchinson, J.N. (1988). Morphology and geotechnical parameters of landslides in relation to geology and hydrogeology. $5^{\text {th }}$ International Symposium on Landslides, Lausanne, Switzerland, vol. 1, 3-35

Jaquet, Y., Duretz, T., Grujic, D., Masson, H. and Schmalholz, S.M. (2018). Formation of orogenic wedges and crustal shear zones by thermal softening, associated topographic evolution and application to natural orogens. Tectonophysics 746, 512-529

Nagel, T.J. and Buck, W.R. (2004). Symmetric alternative to asymmetric rifting models. Geology 32(11), 937-940

Nemcok, M., Mora, A. and Cosgrove, J. (2013). Thick-skindominated orogens: from initial inversion to full accretion: an introduction. Geological Society of London, UK, Special Publications 377, 1- 17

Pasupuleti, V.D.K. (2016). Non-linear numerical modeling of tectonic plate for understanding crustal deformation and stress accumulation at plate junctions. $\mathrm{PhD}$ thesis, International Institute of Information Technology-Hyderabad, India

Platt, J.P. (1986). Dynamics of orogenic wedges and the uplift of high-pressure metamorphic rocks. Geological Society of America Bulletin 97(9), 1037-1053
Ruh, J.B., Kaus, B. J. and Burg, J.P. (2012). Numerical investigation of deformation mechanics in fold-and-thrust belts: Influence of rheology of single and multiple décollements. Tectonics 31(3), TC3005

Sclater, J.G. and Célérier, B. (1987). Extensional models for the formation of sedimentary basins and continental margins. Norsk Geologisk Tidsskrift 67(4), 253-267

Sobolev, S.V. and Babeyko, A.Y. (2005). What drives orogeny in the Andes?. Geology 33(8), 617-620

Sørensen, E.S. (2012). Elasto-plastic hardening Mohr-Coulomb model - Derivation and implementation into the Finite Element Method using principal stress space. Master thesis, Aalborg University, Denmark

Willett, S., Beaumont, C. and Fullsack, P. (1993). Mechanical model for the tectonics of doubly vergent compressional orogens. Geology 21(4), 371-374

Yamato, P., Mouthereau, F. and Burov, E. (2009). Taiwan mountain building: insights from 2-D thermomechanical modelling of a rheologically stratified lithosphere. Geophysical Journal International 176(1), 307-326

Zhang, J. (2014). Numerical modeling of the formation and evolution of basement-involved structures in Wyoming. Doctoral thesis, Rice University, Texas, USA 